\title{
Use of dielectric padding to eliminate low convective field artifact in cr-MREPT conductivity images
}

\author{
Gulsah Yildiz (D) | Yusuf Ziya Ider \\ Department of Electrical and Electronics Engineering, Bilkent University, Ankara, Turkey
}

\section{Correspondence}

Yusuf Ziya Ider, Department of Electrical and Electronics Engineering, Bilkent University, Ankara, Turkey.

Email: ider@ee.bilkent.edu.tr

\section{Funding information}

TUBITAK, Grant/Award Number: 114E522 research grant.
Purpose: Convection-reaction equation-based magnetic resonance electrical properties tomography (cr-MREPT) provides conductivity images that are boundary artifact-free and robust against noise. However, these images suffer from the low convective field (LCF) artifact. We propose to use dielectric pads to alter the transmit magnetic field $\left(\mathrm{B}_{1}{ }^{+}\right)$, shift the LCF region, and eliminate the LCF artifact.

Methods: Computer simulations were conducted to analyze the effects of pad electrical properties, pad thickness, pad height, arc angle, and thickness of the pad-object gap. In 3T MR experiments, water pads and $\mathrm{BaTiO}_{3}$ pads were used with agar-saline phantoms. Two data sets (e.g., with the pad located on the left or on the right of the object [phantom]) were acquired, and the corresponding linear systems were simultaneously solved to get LCF artifact-free conductivity images.

Results: A pad needed to have $180^{\circ}$ arc angle and the same height with the phantom for maximum benefit. Increasing the pad thickness and/or the relative permittivity of the pad increased the LCF shift, whereas excessive amounts of these parameters caused errors in conductivity reconstructions because the effect of neglected $\mathrm{B}_{z}$ terms became noticeable. Conductivity of the pad, on the other hand, had minimal effect on elimination of the LCF artifact. Combining 2 data sets (i.e., with 2 different dielectric pad positions) resulted in more accurate conductivity maps (low $\mathrm{L}^{2}$-errors) as opposed to no pad or single pad cases in experiments and simulations.

Conclusions: Using the proposed technique, LCF artifact is significantly removed, and the reconstructed conductivity values are improved.

\section{1 | INTRODUCTION}

Imaging of the electrical properties (EPs: conductivity, permittivity) of tissues has been found to be useful in many application areas. Conductivity imaging provides clinically important information because of conductivity differences among healthy tissues and also between healthy and malignant tissues. ${ }^{1,2}$ Calculation of specific absorption rate ${ }^{3}$ is another important application in high field MRI. Transcranial magnetic stimulation, ${ }^{4}$ hyperthermia treatment, ${ }^{5}$ and $\mathrm{RF}$ ablation $^{6}$ are examples of therapy monitoring applications requiring EP information.
The idea of calculating EPs at the Larmor frequency of an MR system has been proposed by Haacke in $1991^{7}$ and applied for the first time by Wen in $2003 .{ }^{8}$ Magnetic resonance electrical properties tomography (MREPT) has been reintroduced and extensively analyzed by Katscher in $2009 .^{3}$ MREPT is based on the fact that EPs perturb the RF magnetic field and therefore they can be extracted from the information buried in the RF magnetic field. Haacke has developed the first formula for the relationship between admittivity $\left(\gamma=\sigma+i \omega \varepsilon_{0} \varepsilon_{\mathrm{r}}\right)$ and the RF magnetic field ${ }^{7}$ :

$$
\gamma=\frac{\nabla^{2} \mathrm{~B}_{1}^{+}}{\mathrm{i} \omega \mu_{0} \mathrm{~B}_{1}^{+}}
$$


where $\sigma$ is the conductivity, $\varepsilon_{\mathrm{r}}$ is relative permittivity, $\varepsilon_{0}$ is free-space permittivity, $\omega$ is the Larmor frequency, and $\mathrm{B}_{1}^{+}=\left(\mathrm{B}_{1 \mathrm{x}}+\mathrm{iB}_{1 \mathrm{y}}\right) / 2$ is the transmit magnetic field. ${ }^{3,9}$ Equation 1 will be referred to as the standard MREPT (std-MREPT) method for the reconstruction of EPs. This standard method is point-wise, prone to noise, and more importantly, it assumes locally constant EP values (the so-called local homogeneity assumption [LHA]), which results in error at the tissue boundaries where EPs change abruptly.

Several algorithms that do not use the LHA and that aim at overcoming the boundary artifact issue have been developed. Hafalir et al. ${ }^{10}$ have proposed the cr-MREPT method where EPs are obtained by solving a convection-reaction partial differential equation (PDE) that models the relation between EPs and the $\mathrm{B}_{1}{ }^{+}$field. Gradient-based electrical properties tomography (gEPT) uses a multi-channel transceiver RF coil and obtains the gradients of EPs, which are then integrated starting from a seed-point. ${ }^{11}$ Contrast source inversion (CSI)-based EPT tries to minimize the difference between the measured and the modeled $\mathrm{B}_{1}{ }^{+}$data iteratively to find the EPs. ${ }^{12}$ In Ammari et al., ${ }^{13}$ the forward problem of MREPT is also modeled as a PDE and an inverse problem approach is used to iteratively find the EPs by minimizing the norm of the difference between the measured and calculated $\mathrm{B}_{1}^{+}$. More recently, in Fushimi and Nara ${ }^{14}$ and Nara et al., ${ }^{15}$ a 2 -stage algorithm is developed whereby the axial electric field is also estimated together with the EPs.

Among the several methods, this article focuses on the cr-MREPT algorithm. The cr-MREPT PDE is as follows:

$$
\begin{gathered}
\beta \times \nabla \mathrm{u}+\nabla^{2} \mathrm{~B}_{1}^{+} \mathrm{u}-\mathrm{i} \omega \mu_{0} \mathrm{~B}_{1}^{+}=0 \\
\text { where } \quad \mathrm{u}=\frac{1}{\sigma+\mathrm{i} \omega \varepsilon_{0} \varepsilon_{\mathrm{r}}}, \nabla \mathrm{u}=\left[\begin{array}{c}
\frac{\partial \mathrm{u}}{\partial \mathrm{x}} \\
\frac{\partial \mathrm{u}}{\partial \mathrm{y}} \\
\frac{\partial \mathrm{u}}{\partial z}
\end{array}\right] \text { and } \\
\beta=\left[\begin{array}{c}
\beta_{\mathrm{x}} \\
\beta_{y} \\
\beta_{\mathrm{z}}
\end{array}\right]=\left[\begin{array}{c}
\frac{\partial B_{1}^{+}}{\partial \mathrm{x}}-i \frac{\partial B_{1}^{+}}{\partial \mathrm{y}}+\frac{1}{2} \frac{\partial \mathrm{B}_{\mathrm{z}}}{\partial \mathrm{z}} \\
i \frac{\partial B_{1}^{+}}{\partial \mathrm{x}}+\frac{\partial B_{1}^{+}}{\partial \mathrm{y}}+\frac{i}{2} \frac{\partial \mathrm{B}_{\mathrm{z}}}{\partial \mathrm{z}} \\
\frac{\partial B_{1}^{+}}{\partial \mathrm{z}}-\frac{1}{2} \frac{\partial \mathrm{B}_{z}}{\partial \mathrm{x}}-\frac{i}{2} \frac{\partial \mathrm{B}_{\mathrm{z}}}{\partial \mathrm{y}}
\end{array}\right] .
\end{gathered}
$$

In this PDE, $u$ is the unknown variable. In cylindrical phantoms where there is no change in EPs along z-direction, derivative of $u$ in z-direction becomes 0 . In such cases, the cr-MREPT PDE will simplify to its 2D form, where

$$
\begin{gathered}
\mathbf{F} \times \bar{\nabla} \mathbf{u}+\nabla^{2} B_{1}^{+} \mathrm{u}-i \omega \mu_{0} B_{1}^{+}=0 \\
\bar{\nabla} \mathbf{u}=\left[\begin{array}{c}
\frac{\partial \mathrm{u}}{\partial \mathrm{x}} \\
\frac{\partial \mathrm{u}}{\partial \mathrm{y}}
\end{array}\right] \text { and } \mathbf{F}=\left[\begin{array}{c}
\mathrm{F}_{\mathrm{x}} \\
\mathrm{F}_{y}
\end{array}\right]=\left[\begin{array}{c}
\frac{\partial B_{1}^{+}}{\partial \mathrm{x}}-i \frac{\partial B_{1}^{+}}{\partial \mathrm{y}}+\frac{1}{2} \frac{\partial \mathrm{B}_{z}}{\partial \mathrm{z}} \\
i \frac{\partial B_{1}^{+}}{\partial \mathrm{x}}+\frac{\partial B_{1}^{+}}{\partial \mathrm{y}}+\frac{i}{2} \frac{\partial \mathrm{B}_{z}}{\partial \mathrm{z}}
\end{array}\right]
\end{gathered}
$$

Note that $\mathrm{F}_{\mathrm{y}}=\mathrm{iF}_{\mathrm{x}}$ and $\mathbf{F}$ is referred to as the convective field.

$\mathrm{B}_{\mathrm{Z}}$ cannot be measured in MRI, and using a transverse RF excitation field with a volume birdcage coil, in the center slices, derivatives of $B_{Z}$ are significantly smaller than the derivatives of $\mathrm{B}_{1}{ }^{+}$and, therefore they are neglected by many investigators. The convective field is then calculated as

$$
\mathbf{F}=\left[\begin{array}{c}
\mathrm{F}_{\mathrm{x}} \\
\mathrm{F}_{\mathrm{y}}
\end{array}\right]=\left[\begin{array}{c}
\frac{\partial B_{1}^{+}}{\partial \mathrm{x}}-i \frac{\partial B_{1}^{+}}{\partial \mathrm{y}} \\
i \frac{\partial B_{1}^{+}}{\partial \mathrm{x}}+\frac{\partial B_{1}^{+}}{\partial \mathrm{y}}
\end{array}\right] .
$$

cr-MREPT is a global method, such that it finds the solution for all pixels simultaneously and considers the constraining effects of neighboring pixels on each other, so that it is more robust against noise. However, in regions where $|\mathbf{F}|$ is very low, the solution for $u$ displays artifacts. This region is called the low convective field (LCF) region, and the resulting distortion is called the LCF artifact. ${ }^{10,16}$ Because $F_{y}=\mathrm{iF}_{x}$, we use $F_{x}$ to represent the convective field hereafter. While being a spot-like artifact in the simulation studies, the effect of LCF is increased and widened because of noise in experimental data, resulting in a disturbed region, generally in the center of the object. ${ }^{10}$ gEPT also suffers from a similar artifact, as mentioned as a "global bias" in Lui et al. ${ }^{17}$ It is observed in CSI-based methods that low E field regions, which are identical with the LCF regions, result in artifacts. ${ }^{12,18}$ Recent MREPT algorithms such as in Ammari et al., ${ }^{13}$ Fushimi and Nara ${ }^{14}$ and Nara et al. ${ }^{15}$ also suffer from artifacts in the LCF region.

There have been suggestions for segmenting out the LCF regions to get rid of the secondary effects of this artifact ${ }^{10,13}$ and use std-MREPT in those regions. This suggestion does not potentially work when LCF coincides with tissue boundaries. Other suggestions for LCF artifact elimination have been based on double- (and multi-) excitations. The purpose is to modify the $\mathrm{B}_{1}{ }^{+}$field in such a way that the LCF region is displaced in different excitations, and data from all excitations are used to mitigate the LCF artifacts. One type of multi-excitation is based on modifying the RF field by coil manipulations. This kind of double-excitation was originally proposed in Zhang et al. ${ }^{19}$ (a starting study that later led to the multi-channel-based methods like those in Liu et al. ${ }^{11}$ ). Although explicit reference was not made to LCF artifact in Zhang et al., ${ }^{19}$ stripe-like artifacts were observed in EP reconstructions concomitant with the 2 orthogonal linear RF excitations used via a birdcage coil. A dual-excitation method is recently presented by Ariturk and Ider, ${ }^{16}$ where a multichannel TEM array is used to obtain different $\mathrm{B}_{1}{ }^{+}$fields with shifted LCF regions. This method, however, is demanding on the RF amplifiers and requires a multi-transmit coil and system. A second type of multi-excitation is based on using pads 
to alter the RF field in the object. Although high dielectric pads were already being used in high field MRI for shimming purposes, ${ }^{20-23}$ padding was applied for the first time in simulation studies to improve EP estimation in a CSI-EPTbased method. ${ }^{18}$ In this study, 2 scans, with and without high permittivity pad placed near the object, are used to obtain EP estimations corresponding to 2 different electric field distributions and hence to 2 different LCF regions. These 2 estimations are then averaged to improve the EP maps. Hafalir et al. ${ }^{10}$ proposed a double-excitation method by cutting a portion of the phantom and repeating the data collection. Although it eliminates the LCF artifact, in their cr-MREPT algorithm, it would be impractical in a real-life application. In a preliminary study, ${ }^{24}$ we have also shown that using high dielectric pads with cr-MREPT, one can shift the LCF region.

Gurler and $\operatorname{Ider}^{25}$ proposed a phase-based cr-MREPT method, which uses the $\mathrm{B}_{1}{ }^{+}$phase data only and suffers less from LCF artifact. In general, phase-based methods give high contrast conductivity images, but they fail to give the correct values as they assume low $\mathrm{B}_{1}{ }^{+}$magnitude gradients. ${ }^{26,27}$ Regularization, based on introducing an artificial diffusion term in the cr-MREPT PDE, has been proposed to mitigate LCF artifacts. ${ }^{28,29}$ Determination of the value of the regularization parameter (the diffusion constant) is still a major issue in such methods because one has to compromise spatial resolution (concomitant with blurring) with elimination of the LCF artifact. Although one may experience complete elimination of the LCF artifact in some numerical simulation cases, it is our experience that LCF artifact reappears when noise is added to the simulated data or when actual noisy experimental data are used. ${ }^{29}$

In this article, we investigate the double-excitation method using dielectric pads to eliminate the LCF artifact in cr-MREPT conductivity maps. First, the effect of a dielectric pad on the $\mathrm{B}_{1}{ }^{+}$field in the object is studied by simulations, and then padding is applied both in simulation and experimental studies. Data from 2 cases, for which the LCF regions do not overlap, are used to solve the corresponding PDEs simultaneously to reconstruct conductivity using the cr-MREPT method. Results for different pads are simulated and the optimum pad structure for MREPT is determined. Phantom experiments with $\mathrm{BaTiO}_{3}$ slurry pads and water pads are also conducted, and the reconstructed conductivity images are compared.

\section{2 | METHODS}

\section{1 | Simulation methods}

We conducted simulations in COMSOL Multiphysics (COMSOL AB, Stockholm, Sweden) using the frequency domain study in radio frequency module. Quadrature birdcage coil model was used for transmission. The coil was 24 $\mathrm{cm}$ in height, $14.5 \mathrm{~cm}$ in radius, and had 16 rungs. The coil was excited in the quadrature volume transmit mode where 2 ports that were spatially $90^{\circ}$ apart were driven by voltage sources $(100 \mathrm{~V} \mathrm{rms})$ with $90^{\circ}$ phase offset with respect to each other. ${ }^{30}$ Electromagnetic study was performed at 127.7 $\mathrm{MHz}$, the nominal frequency of a 3T MR system. Calculated $\mathrm{B}_{1}{ }^{+}$was exported with $1 \mathrm{~mm}$ resolution.

\subsection{1 | Simulation phantoms and pads}

A cylindrical phantom (height $=15 \mathrm{~cm}$, radius $=6 \mathrm{~cm}$ ) with 2 anomalies was designed (Figure 1A). Small anomaly had $\sigma=1 \mathrm{~S} / \mathrm{m}$ and large anomaly had $\sigma=1.5 \mathrm{~S} / \mathrm{m}$, whereas background had $\sigma=0.5 \mathrm{~S} / \mathrm{m}$. The whole phantom had $\varepsilon_{\mathrm{r}}=80$ and $\mu_{\mathrm{r}}=1$ (relative permeability). A 3D tetrahedral mesh was used for COMSOL simulations. For $-0.5 \mathrm{~cm}<\mathrm{z}<0.5$ $\mathrm{cm}$, the mesh size was $<1.75 \mathrm{~mm}$, and the data were taken from the $\mathrm{z}=0$ slice. Mesh size was $3 \mathrm{~mm}$ at most in the rest of the phantom (Figure 1B). In some studies, the anomalies were removed and a homogeneous phantom with $\varepsilon_{\mathrm{r}}=80$ and $\sigma=0.5 \mathrm{~S} / \mathrm{m}$ was obtained. Figure 1D displays a 3D head model,${ }^{30}$ the conductivity properties of which are shown in Figure 1F. Mesh was arranged similar to the cylindrical phantom (Figure $1 \mathrm{E}$ ), and the data were taken from the $\mathrm{z}=0$ slice.

In Figure 1A-B, an example pad with $1 \mathrm{~cm}$ thickness and $2 \mathrm{~mm}$ gap is shown, where it lies along the full height of the object. When pure water pads were simulated, the corresponding material properties were $\varepsilon_{\mathrm{r}}=80$ and $\sigma=0 \mathrm{~S} / \mathrm{m}$. The higher relative permittivity values (e.g., 150, 220, and 290) are meant to represent pads made by different ratios of $\mathrm{BaTiO}_{3}$ and water. Pads used for simulation cylindrical phantoms had pad angle (defined in Results section) of $180^{\circ}$ and pad height of $15 \mathrm{~cm}$ unless specified otherwise. Pad in the head model simulation is shown in Figure 1D. Head pad was designed to have a shape that would be expected in a real experiment.

\section{2 | Experimental methods}

\subsection{1 | Experimental phantom preparation}

Cylindrical experimental phantom (height $=17 \mathrm{~cm}$, radius $=$ $6.25 \mathrm{~cm}$ ) was used. Background of the phantom was prepared using an agar-saline gel $(20 \mathrm{~g} / \mathrm{L}$ agar, $2 \mathrm{~g} / \mathrm{L} \mathrm{NaCl}, 1.5 \mathrm{~g} / \mathrm{L}$ $\mathrm{CuSO}_{4}$ ), and the higher conductive regions were prepared using a saline solution $(20 \mathrm{~g} / \mathrm{L}$ agar, $6 \mathrm{~g} / \mathrm{L} \mathrm{NaCl}, 1.5 \mathrm{~g} / \mathrm{L}$ $\mathrm{CuSO}_{4}$ ). Background was expected to have $\sim 0.5 \mathrm{~S} / \mathrm{m}$ conductivity where the anomaly regions were expected to have $\sim 1 \mathrm{~S} / \mathrm{m}^{31}$

\subsection{2 | Pad preparation}

$\mathrm{CaTiO}_{3}$ and $\mathrm{BaTiO}_{3}$ are the most widely used materials for padding because of their high dielectric constants. ${ }^{32}$ The 
powder form of these materials are mixed with water to obtain slurries, while a bead-water mixture can also be used. ${ }^{33}$ In this study, 2 kinds of material for padding were considered: water and $\mathrm{BaTiO}_{3}$ slurry. Two different $\mathrm{BaTiO}_{3}$ powders from different vendors (MERCK, Darmstadt, Germany and Entekno, Eskisehir, Turkey) were used, 2 slurries with $\mathrm{BaTiO}_{3}$-water weight ratio of 2-1 were prepared. The slurries were collected into polyethylene bags that were heatsealed (Figure 1G).

Std-MREPT was used to measure the dielectric constant and the conductivity of the resulting slurries. Because the $\mathrm{BaTiO}_{3}$ slurry (suspension) was not homogenous and also some of the $\mathrm{BaTiO}_{3}$ precipitated in time, std-MREPT images were noisy and the obtained $\mathrm{B}_{1}{ }^{+}$needed to be highly filtered. A $5 \times 5 \times 5$ median filter and $5 \times 5 \times 5$ Gaussian filter with $\mathrm{SD}$ of 5 were applied. For 2 different $\mathrm{BaTiO}_{3}$ slurries, dielectric constant and conductivity were obtained as: for Merck, $\varepsilon_{\mathrm{r}}=187.6 \pm 42.5(\mathrm{SD})$ and $\sigma=0.05 \mathrm{~S} / \mathrm{m} \pm 0.8(\mathrm{SD})$; for Entekno, $\varepsilon_{\mathrm{r}}=214.1 \pm 18.9(\mathrm{SD})$ and $\sigma=1.96 \mathrm{~S} / \mathrm{m} \pm 0.45$ (SD). These $\mathrm{BaTiO}_{3}$ pads were later referred to as the nonconductive $\mathrm{BaTiO}_{3}$ slurry pad and the conductive $\mathrm{BaTiO}_{3}$ slurry pad, respectively.

\subsection{3 | Experiment setup and registration of data sets}

In general, 3 consecutive data sets were acquired: without pad (NP-no pad), pad on the left side (LP), and pad on the right side (RP). One important point was that the object should not move between the successive experiments. A Styrofoam dock was used to prevent motion during the experiment (Figure 1H). However, while placing and stabilizing the pad, the phantom still had some displacement. To spatially match the data sets, "Genetic Algorithm" (GA) method was used as
(A)

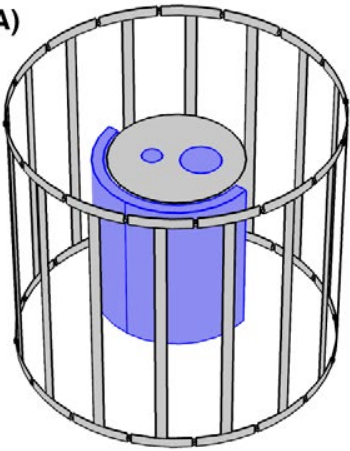

(D)

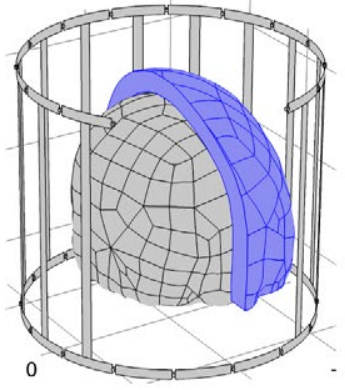

(B)

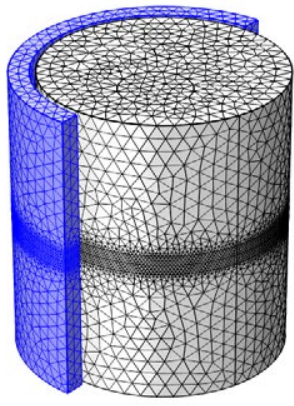

(E)

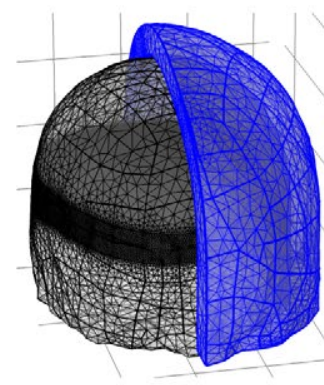

(C)

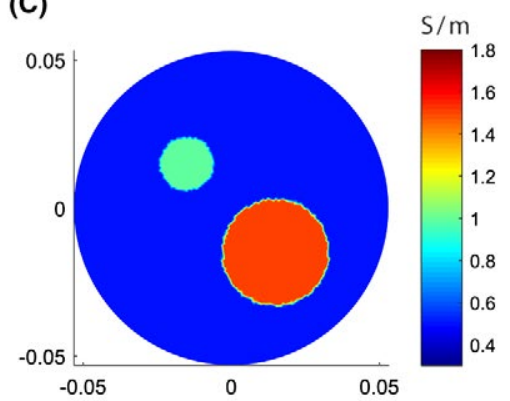

(F)

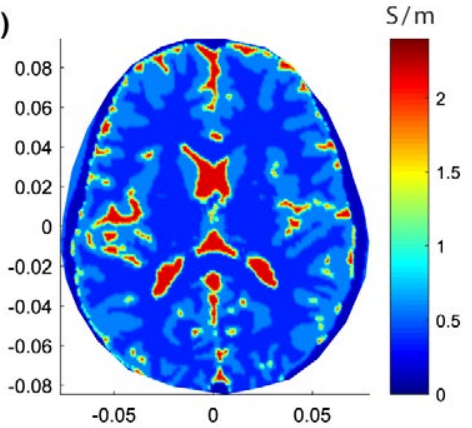

(G)

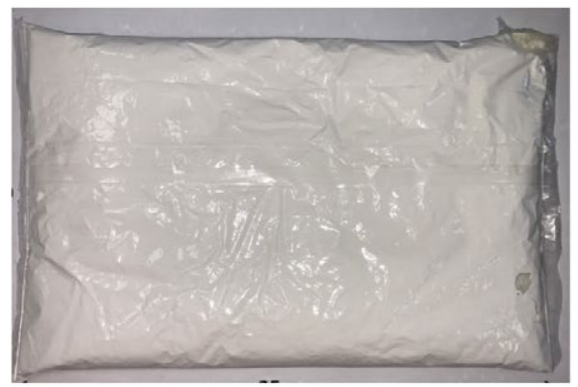

(H)

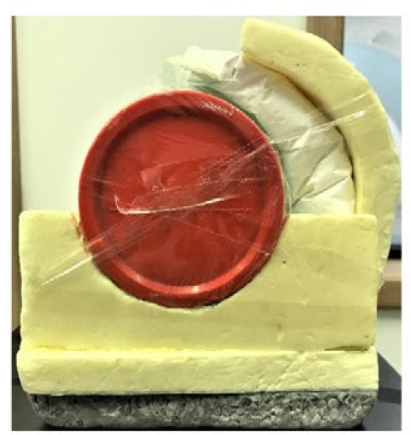

F I G U RE 1 Simulation and experimental phantoms and pads. (A) The birdcage coil, cylindrical phantom, and the pad. The pad and the anomaly regions are shown with blue. (B) Mesh used for the cylindrical phantom. (C) Conductivity values assigned to the cylindrical phantom. (D) The birdcage coil, the head phantom, and the pad. (E) Mesh used for the head phantom. (F) Conductivity values assigned to the head phantom at the $\mathrm{z}=0$ slice. $(\mathrm{G}) \mathrm{A} \mathrm{BaTiO}_{3}$ slurry pad. (H) An experimental setup with the phantom, the pad, and the Styrofoam dock 
the optimization tool for registration of the images onto each other. $^{34}$

\subsection{4 | MR sequences}

Experiments were performed using Siemens Tim Trio 3T Scanner (Erlangen, Germany). We used double angle method ${ }^{35}$ for $\mathrm{B}_{1}^{+}$magnitude mapping and balanced steadystate free precession (bSSFP) sequence to obtain the $\mathrm{B}_{1}^{+}$ phase. ${ }^{36}$ Quadrature birdcage body coil was used for transmit, and phased-array (12 channel head matrix coil, Siemens) was used for receive in the 2 gradient-echo sequences that are necessary for double angle method. Sequence parameters were as follows: field of view $=170 \mathrm{~mm}$, voxel size $=$ $1.3 \mathrm{~mm} \times 1.3 \mathrm{~mm} \times 3 \mathrm{~mm}$, flip angles $=60 / 120, \mathrm{TE} / \mathrm{TR}=$ $5 / 1500 \mathrm{~ms}$, number of excitations $=4$, total duration for double angle method $=26 \mathrm{~min}$.

bSSFP was used because of its speed and high SNR features. It does not have the additional phase component because of eddy-currents, which makes it a better option than a spin-echo sequence. ${ }^{36}$ Body coil was used both for transmit and receive. Transceive phase approximation (TPA) was used, and the transmit phase was taken as half of the transceive phase. ${ }^{3}$ bSSFP parameters were: field of view $=170$ $\mathrm{mm}$, voxel size $=1.3 \mathrm{~mm} \times 1.3 \mathrm{~mm} \times 3 \mathrm{~mm}$, flip angle $=$ $40^{\circ}, \mathrm{TE} / \mathrm{TR}=2.23 / 4.46 \mathrm{~ms}$, number of excitations $=32, \mathrm{du}-$ ration $=20 \mathrm{~s}$.

\section{3 $\quad$ Numerical methods}

Numerical methods were implemented in MATLAB (The MathWorks, Natick, MA). Either from the simulation environment or from the MRI experiments, $\mathrm{B}_{1}{ }^{+}$was obtained on a regular grid and interpolated into a 2D triangular mesh. For experiments, a diffusion filter, which corresponds to a Gaussian filter with SD of $1.7 \mathrm{~mm}$, was used for denoising, using MATLAB'S PDE toolbox. Gradients and Laplacian were calculated using the method proposed by Fernandez. ${ }^{37}$

The cr-MREPT PDE was discretized to build a linear system of equations as explained in Hafalir et al. ${ }^{10}$ At the object boundaries, we used Dirichlet boundary condition, and boundary values were set to $\sigma=0.5 \mathrm{~S} / \mathrm{m}$ and $\varepsilon_{\mathrm{r}}=80$. Even if the given boundary values may not be exactly correct, the values converge to the correct ones within a couple of pixels toward the inside of the object. While finding $u$, backslash operator of MATLAB was used, which uses the minimum norm least-squares approach. When the 2 data sets, or more, were being used simultaneously, the system of equations corresponding to different data sets were concatenated, and again the backslash operator was used. We use the term "combined conductivity" when conductivity is reconstructed using 2 or more data sets as explained.

\section{3 | RESULTS}

\section{1 | Simulation results}

\subsection{1 | Effect of pad on the RF transmit field $\mathbf{B}_{1}^{+}$}

We conducted simulations to understand and visualize the effect of the pad on the object. The primary rotating electromagnetic field created by the birdcage coil results in current flow within both the pad and the object. The homogeneous phantom together with a (left) pad that has uniform EP of $\varepsilon_{\mathrm{r}}=150$ and $\sigma=0 \mathrm{~S} / \mathrm{m}$ were simulated, and the current distribution in the pad is displayed in Figure 2A. At different phase instants, current flow direction and intensity changed throughout the pad as shown in Figure 2A-D. Also an animation showing the rotating current distribution is provided by the Supporting Information Video S1.

One can view the current in the pad as a source that can generate an additional $\mathrm{B}_{1}^{+}$field in the object. To exhibit its contribution (i.e., the effect of the pad on $\mathrm{B}_{1}{ }^{+}$), we conducted 3 simulations with the homogeneous phantom using a left pad with $3 \mathrm{~cm}$ thickness and with EPs of $\varepsilon_{\mathrm{r}}=150$ and $\sigma=0 \mathrm{~S} / \mathrm{m}$

1. The birdcage coil was excited but a pad was not introduced. The transmit magnetic field generated in the object (i.e., the field caused by the coil) is denoted as $\mathrm{B}_{\mathrm{C}}{ }^{+}$.

2. The birdcage coil was excited, and a pad was placed on the left-hand side of the object. The transmit field for this simulation, is called $\mathrm{B}_{\mathrm{T}}{ }^{+}$( $\mathrm{T}$ for total). The current distribution induced in the pad was saved to disc.

3. The birdcage coil was not excited (i.e., the driving voltage sources were killed) and the pad was replaced by a volume current source identical to the current distribution saved in the previous step. The field in the object obtained in this case (i.e., the contribution of the pad to the object's transmit field) is called $\mathrm{B}_{\mathrm{P}}{ }^{+}$.

Magnitudes of $\mathrm{B}_{\mathrm{C}}{ }^{+}, \mathrm{B}_{\mathrm{P}}{ }^{+}$, and $\mathrm{B}_{\mathrm{T}}{ }^{+}$, as well as the magnitude of $\left(\mathrm{B}_{\mathrm{C}}^{+}+\mathrm{B}_{\mathrm{p}}^{+}\right)$, are shown in Figure $2 \mathrm{E}-\mathrm{H}$. It is observed that $\left|\mathrm{B}_{\mathrm{C}}^{+}+\mathrm{B}_{\mathrm{P}}^{+}\right| \approx\left|\mathrm{B}_{\mathrm{T}}^{+}\right|$as expected. With the introduction of the pad, the transmit field close to the dielectric pad became higher in magnitude as compared to the case without pad. We repeated the same simulation set with a pad that additionally had conductive property $\left(3 \mathrm{~cm}\right.$ thick and with EPs of $\varepsilon_{\mathrm{r}}=150$ and $\sigma=2$ $\mathrm{S} / \mathrm{m})$, and the same transmit fields as explained above are shown in Figure 2I-L. In this case, with the introduction of the pad, the total transmit field in the object decreases somewhat in magnitude and shifts along a different direction than with the pure dielectric pad. 
(A)

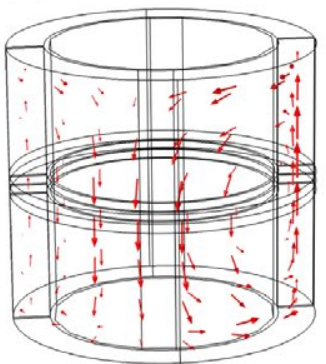

(E) $\left|\mathrm{B}_{\text {Coil }}{ }^{+}\right|$

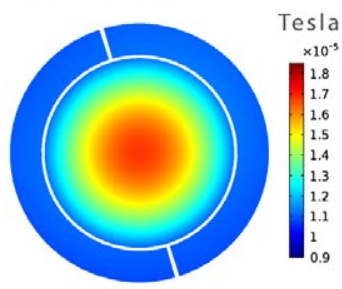

(G) $\left|\mathrm{B}_{\text {Total }}{ }^{+}\right|$

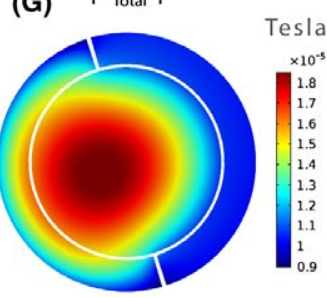

(B)

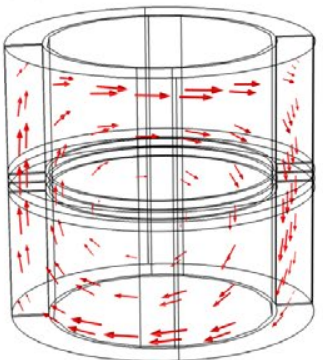

(F) $\mid \mathrm{B}_{\mathrm{Pad}^{+}}{ }^{+}$

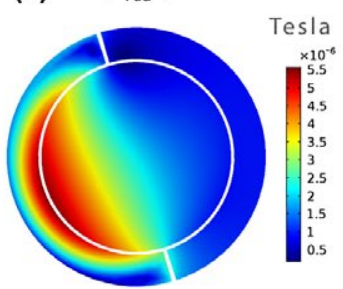

(H) $\left|\mathrm{B}_{\text {Coil }}{ }^{+}+\mathrm{B}_{\text {Pad }}{ }^{+}\right|$

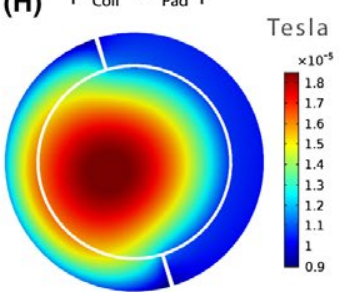

(C) $180^{\circ}$

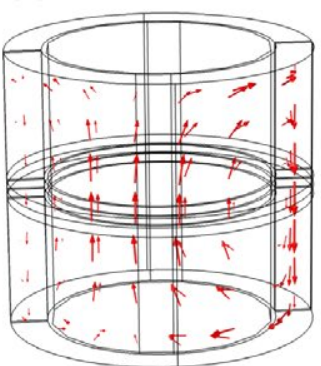

(l)

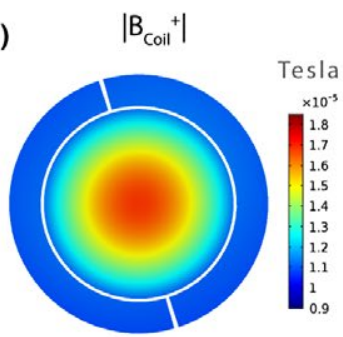

(K)

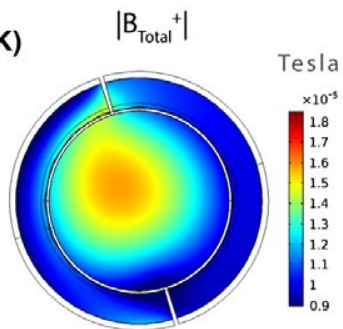

(D)

$270^{\circ}$
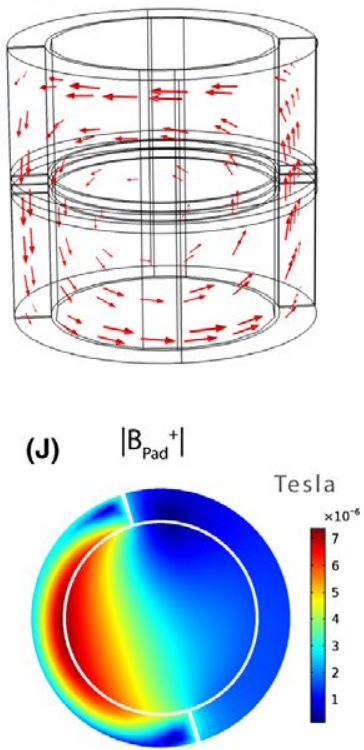

(L)

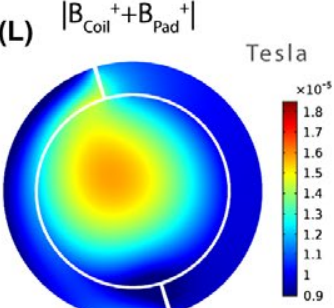

F I G URE 2 Effect of the left pad on the $\mathrm{B} 1^{+}$magnitude and the current distribution in the pad. (A-D) Current distribution in the pad $(\epsilon=150, \sigma=0 \mathrm{~S} / \mathrm{m}$, pad thickness $=3 \mathrm{~cm}$ and gap thickness $=2 \mathrm{~mm})$ at $0^{\circ}, 90^{\circ}, 180^{\circ}$, and $270^{\circ}$ phase instances, respectively. (E-H) Magnitudes of $\mathrm{B}_{\mathrm{C}}{ }^{+}, \mathrm{B}_{\mathrm{P}}{ }^{+}, \mathrm{B}_{\mathrm{T}}{ }^{+}$, and $\mathrm{B}_{\mathrm{C}}{ }^{+}+\mathrm{B}_{\mathrm{P}}{ }^{+}$, respectively, for the same pad. (I-L) Magnitudes of $\mathrm{B}_{\mathrm{C}}{ }^{+}, \mathrm{B}_{\mathrm{P}}{ }^{+}, \mathrm{B}_{\mathrm{T}}{ }^{+}$, and $\mathrm{B}_{\mathrm{C}}{ }^{+}+\mathrm{B}_{\mathrm{P}}{ }^{+}$, respectively, for another pad $(\epsilon=150, \sigma=2 \mathrm{~S} / \mathrm{m}$, pad thickness $=3 \mathrm{~cm}$, and gap thickness $=2 \mathrm{~mm})$. Subscripts C, P, T are for "Coil," "Pad," and "Total," respectively

\subsection{2 | Effect of pad parameters on LCF shift}

The amount of the LCF shift in the presence of a pad depends in general on the amount of current flowing inside the pad. EPs of the pad material, thickness of the pad (PT), angle of the arc that the pad subtends (PA), the height of the pad (PH), and the gap thickness (GT) were considered to be effective on the amount of current, and they were investigated using simulations.

For the no-pad case, the LCF region is almost at the center (Figure 3B) and the location of the minimum value of the convective field is shown in Figure 3C. We placed a left-hand side pad (PT $=2 \mathrm{~cm}, \mathrm{GT}=2 \mathrm{~mm}, \sigma=0 \mathrm{~S} / \mathrm{m})$ and varied its dielectric constant; the corresponding convective field minimums are displayed in Figure 3D. The direction of the LCF shift was toward one end of the pad; moreover, for a fixed pad location, changing the pad's dielectric constant, thickness, height, and the gap thickness did not alter the direction of the shift but only the amount of it. However, looking at Figure $3 \mathrm{E}$, this is not the case for changing the pad's conductivity.
Keeping other parameters fixed and varying the conductivity of the pad, one can observe that the convective field minimums shift almost on an arc centered on the "no-pad (without pad) minimum location" rather than on a straight trajectory. A similar difference arose with the $\mathrm{B}_{1}{ }^{+}$magnitudes given in Figure 2, such that the behavior of $\mathrm{B}_{1}^{+}$was also different between pure-dielectric and conductive-dielectric pads.

Figure 4A-D4 display the dependence of the amount of LCF shift on the PT, the GT, and the dielectric constant $\left(\varepsilon_{\mathrm{r}}\right)$ of the pad. We calculated the amount of LCF shift as the Euclidean distance between the convective field minimums of the cases no-pad and a left pad. No-gap pads (GT $=0 \mathrm{~mm}$ ) yielded rather high shifts than pads with non-zero gap. The presence of a gap introduces a very low dielectric medium (air) between the pad and the object, and consequently the effect of the pad is significantly reduced. However, in practice, because a gap may be unavoidable, it is more interesting to observe the results of a non-zero gap and even up to $30 \mathrm{~mm}$ shifts are possible with such pads. One can recognize that, for a $\mathrm{PT}=1$ $\mathrm{cm}$ and GT $=2 \mathrm{~mm}$ pad, $3-7 \mathrm{~mm}$ shifts are possible as $\varepsilon_{\mathrm{r}}$ is varied between 80 and 290. We achieved similar amount of 

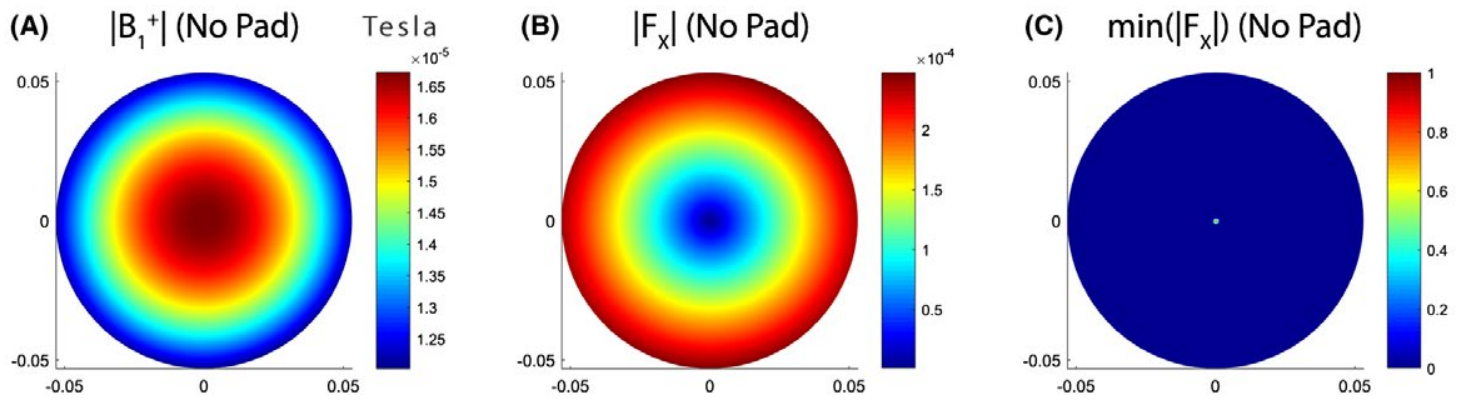

(D)

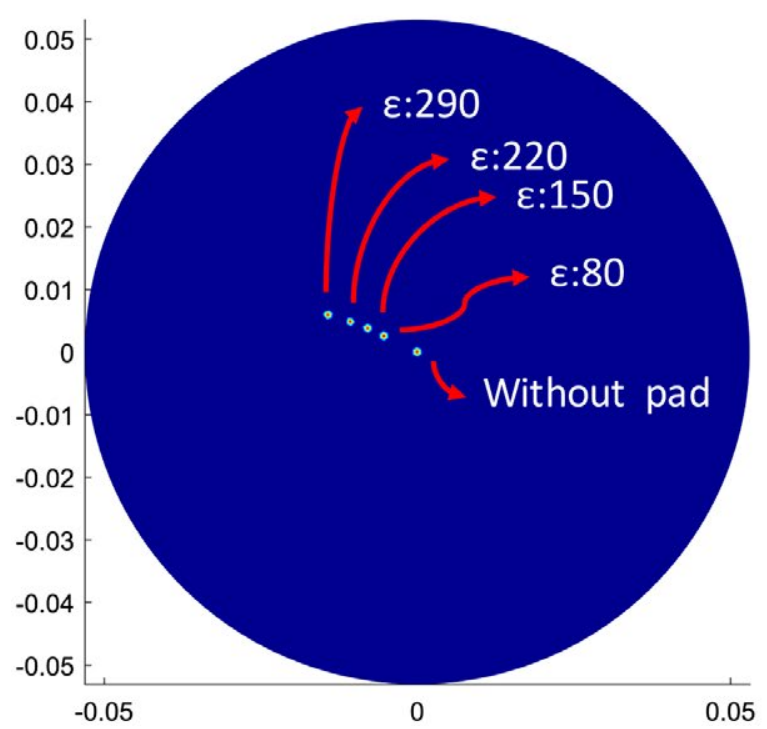

(E) $\min \left(\left|F_{x}\right|\right)$ (varying pad conductivity)

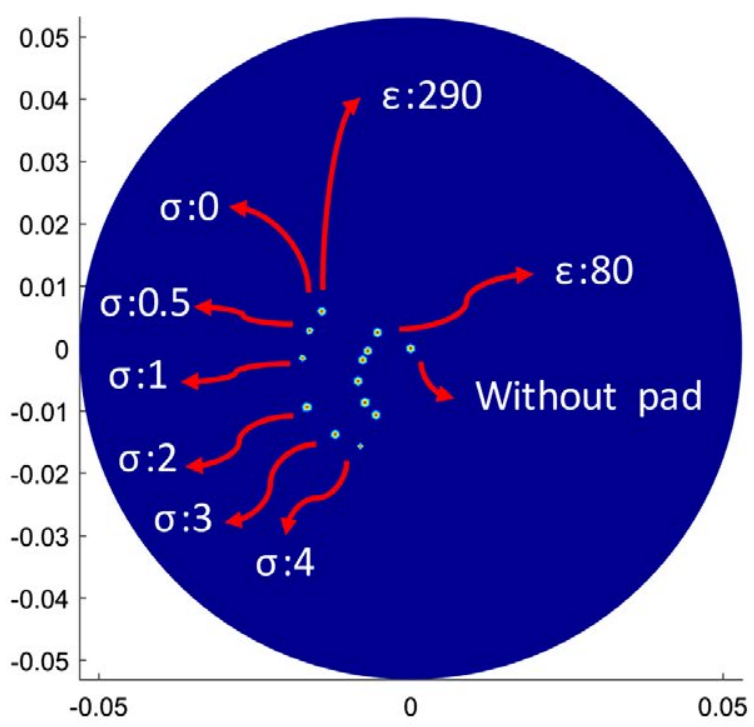

F I G URE 3 Effect of the EPs of the pad on the LCF shift. For homogeneous phantom without a pad, (A) magnitude of $\mathrm{B}_{1}{ }^{+}$, (B) magnitude of the convective field $\left(\left|F_{X}\right|\right)$, and $(C)$ location of the minimum value of $\left|F_{X}\right|$. (D) Locations of the minimum values of $\left|F_{X}\right|$ formed by the pads with different $\epsilon_{\mathrm{r}}$. (E) Locations of the minimum values of $\left|\mathrm{F}_{\mathrm{X}}\right|$ formed by the pads with different $\sigma(\mathrm{S} / \mathrm{m})$; inner points are for $\epsilon_{\mathrm{r}}=80$ and the outer points are for $\epsilon_{\mathrm{r}}=290$ pads

shifts with a 2-cm thick pad even when GT is $8 \mathrm{~mm}$. In general, looking through the different PT results, if GT is needed to be increased, then the PT can be increased to balance the amount of the shift. Considering the effect of $\varepsilon_{\mathrm{r}}$, the amount of the shift increased with increasing dielectric constant, irrespective of the values of the other parameters. Dependence of LCF shift to $\varepsilon_{\mathrm{r}}$ seems to be linear with $\mathrm{PT}=1$ and $2 \mathrm{~cm}$ pads, but the incremental effect was more pronounced as $\varepsilon_{\mathrm{r}}$ was increased for the cases of $\mathrm{PT}=3$ and $4 \mathrm{~cm}$ pads. LCF shift dependence on 1/GT, on the other hand, was not linear, in the sense that doubling GT did not cause the amount of the shift to be halved. Increasing PT or $\varepsilon_{\mathrm{r}}$ both acted to increase the LCF shift and therefore, they can be used as a substitute for each other. For example, a pad with $\mathrm{PT}=2 \mathrm{~cm}$ and $\varepsilon_{\mathrm{r}}=290$, and another pad with $\mathrm{PT}=4 \mathrm{~cm}$ and $\varepsilon_{\mathrm{r}}=150$, both caused $\sim 15 \mathrm{~mm}$ of LCF shift.

Another set of simulations was performed to clarify the effect of the angle that the pad subtends (PA). PT $=3 \mathrm{~cm}$ pad with $\varepsilon_{\mathrm{r}}=150$, and GT $=2 \mathrm{~mm}$ was wrapped around the phantom with increments of $45^{\circ}$ until it reached full coverage.
Until $180^{\circ}$ of PA, the amount of LCF shift increased, whereas after $180^{\circ}$, the effect reversed and the amount of the shift decreased, because the effects enforced from opposite sides began to cancel (Figure 4E).

We also varied the height of the pad (PT $=3 \mathrm{~cm}$, GT = $2 \mathrm{~mm}, \varepsilon_{\mathrm{r}}=150$ ) with $2.5-\mathrm{cm}$ increments while keeping the center of the pad fixed at $\mathrm{z}=0$. Until $15 \mathrm{~cm}$, which was also the height of the object itself, the relation seemed to be almost linear (Figure 4F). When PH exceeded the height of the object, increments in the shifts got smaller, although the LCF still shifted further.

\subsection{3 | Effect of pad parameters on combined conductivity maps}

To monitor the effect of the pad parameters on the final conductivity map, we separately reconstructed the individual pad cases and also reconstructed the combined cases, in which the first phantom model with 2 anomaly regions was used. 
(A) LCF Shift vs Dielectric Constant

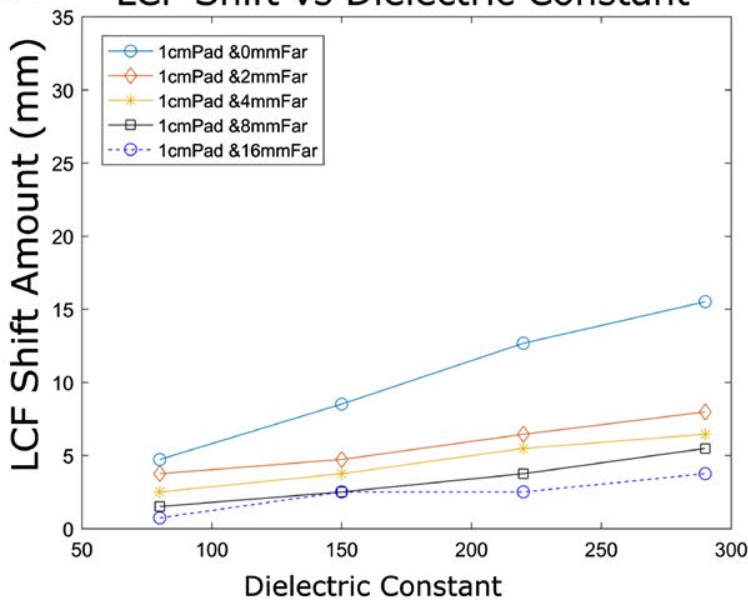

(C) LCF Shift vs Dielectric Constant

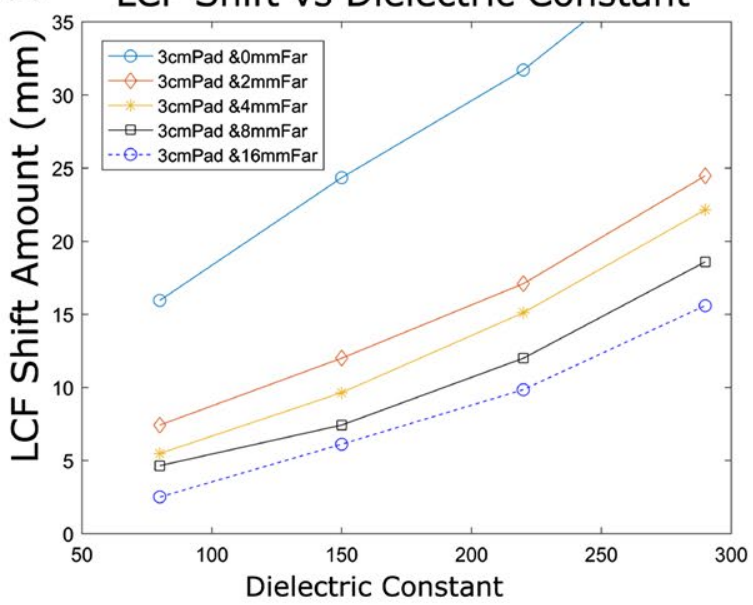

(E) LCF Shift vs Pad Arc Angle (PA)

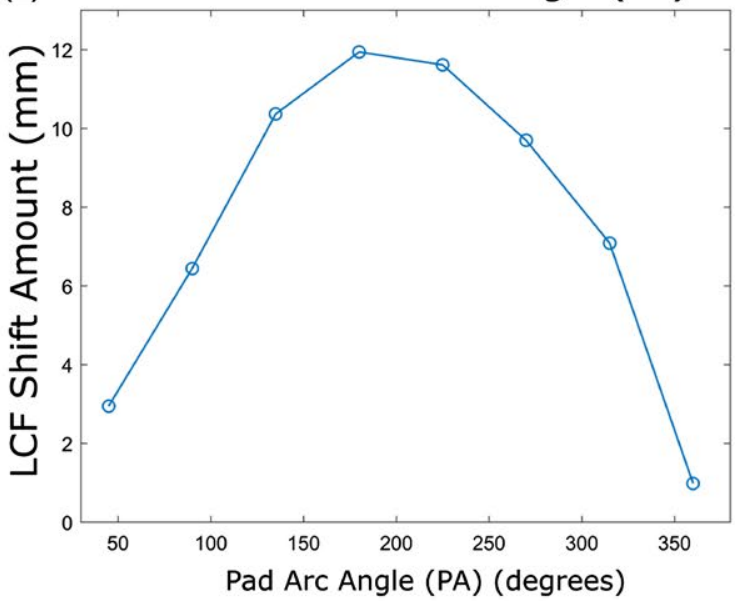

(B) LCF Shift vs Dielectric Constant

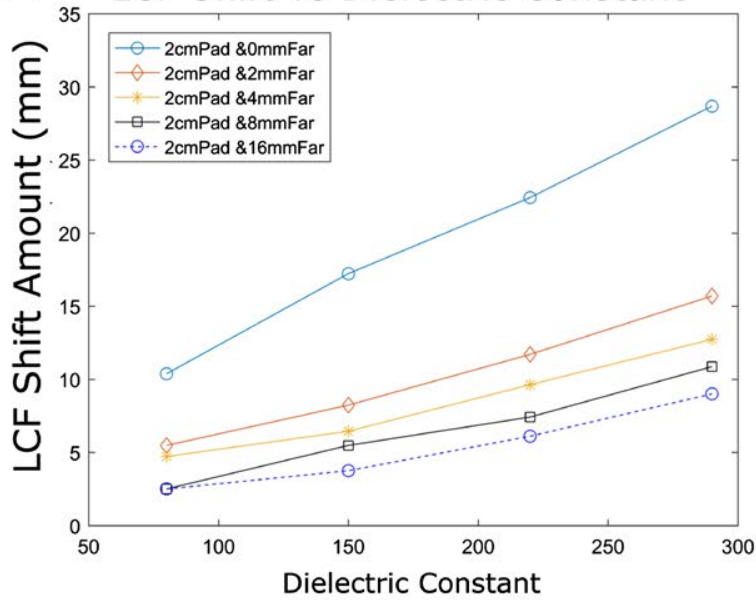

(D) LCF Shift vs Dielectric Constant

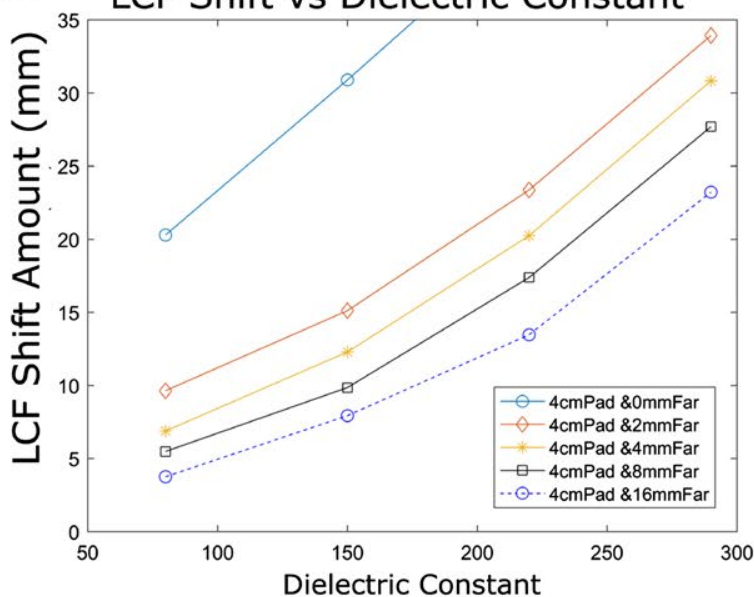

(F) LCF Shift vs Height of the Pad (PH)

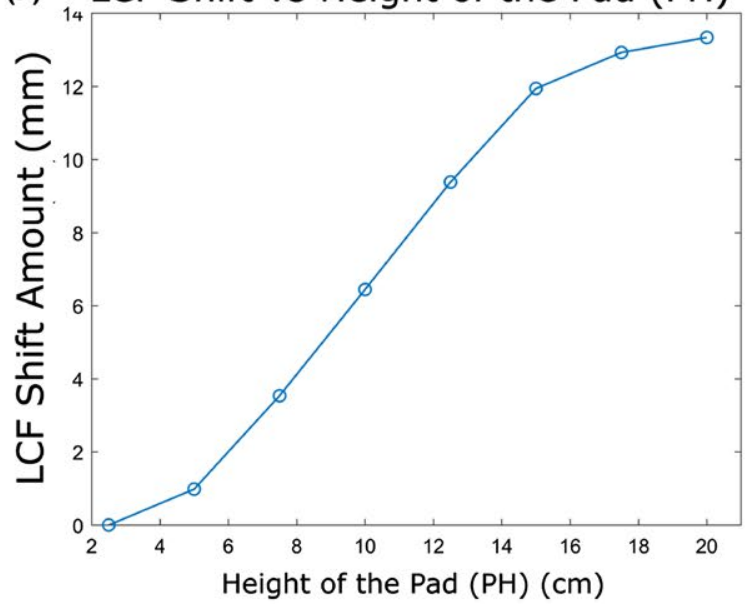

F I G U R E 4 Effects of pad parameters on the LCF shift. LCF shift versus dielectric constant of the pad for: (A) pad thickness (PT) $=1 \mathrm{~cm}$, (B) pad thickness $=2 \mathrm{~cm},(\mathrm{C})$ pad thickness $=3 \mathrm{~cm}$, and (D) pad thickness $=4 \mathrm{~cm}$. Results for different gap thicknesses (GT) are shown on the same graphs. (E) LCF shift versus pad angle (PA). (F) LCF shift versus pad height (PH)

Dielectric constant of 290 is a relatively high value and the highest that we analyzed, and the usage of such high permittivity pad $(\mathrm{PT}=1 \mathrm{~cm})$ succeeded to separate LCF regions of different cases from each other to a large extent (Figure 5D-F). Examining the behavior of LCF artifacts in conductivity reconstructions shown in Figure 5G-I, it is observed that LCF artifacts did not present themselves in a predetermined shape, but instead changed their pattern depending on whether the LCF was in or out of a conductivity anomaly or whether it coincided with the boundary of an 

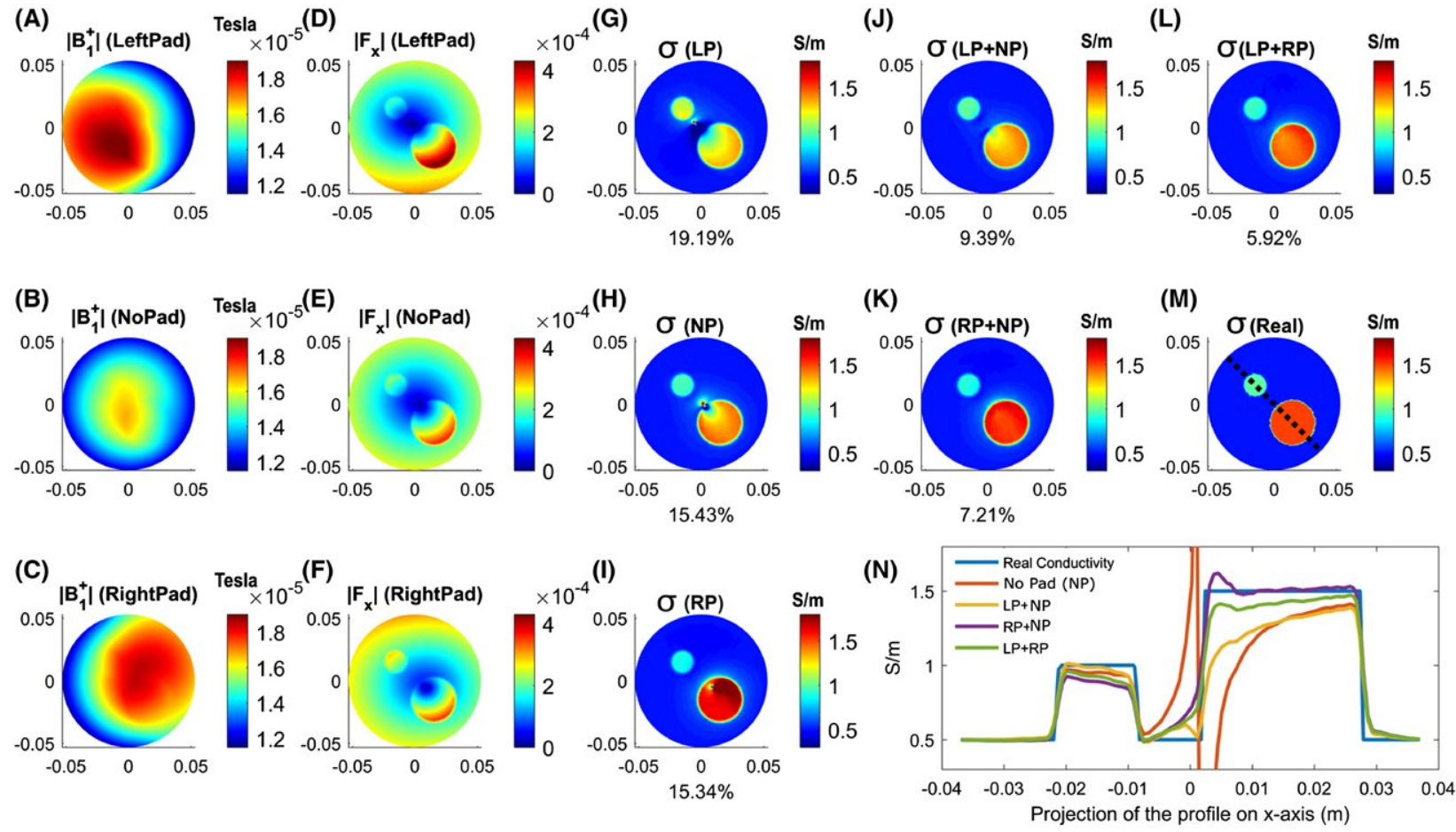

F I G U RE 5 Simulation results with the $\epsilon=290, \sigma=0 \mathrm{~S} / \mathrm{m}$, pad thickness $=1 \mathrm{~cm}$, and gap thickness $=2 \mathrm{~mm}$ pad. (A-C) Magnitude of $\mathrm{B}_{1}{ }^{+}$. (D-F) Magnitude of $F_{X} \cdot(G-I)$ Reconstructed conductivity image for left-pad (LP), no-pad (NP), and right-pad (RP), respectively. (J) Reconstructed conductivity image for LP + NP combination. (K) Reconstructed conductivity image for RP + NP combination. (L) Reconstructed conductivity image for LP + RP combination. (M) Real conductivity map. (N) Conductivity profiles on the dashed line in (M). Percent $\mathrm{L}^{2}$-errors are given below the figures

anomaly; the LCF artifact had patterns like a single dip, a single peak, or both, with the effect fading within couple of pixels or within dozens.

As it was proposed, different data sets were combined to get rid of the LCF artifact: left pad and no-pad (LP + NP), right pad and no-pad (RP + NP), and left pad and right pad (LP $+\mathrm{RP}$ ). Figure 5J-L display the corresponding combined conductivity results, and the conductivity profiles (on the dashed line given in Figure $5 \mathrm{M}$ ) are plotted in Figure 5N. Percent $\mathrm{L}^{2}$-errors (calculated excluding the anomaly boundaries) for each conductivity reconstruction are also provided below the corresponding reconstruction. LP + RP combination gave better accuracy and it effectively eliminated the LCF artifact. A similar set of simulation results were provided in Supporting Information Figure S1 for the same pad but with $\varepsilon_{\mathrm{r}}=80$. Looking at the LCF artifacts in cases with and without pads, they were not sufficiently separated from each other (the LCF regions overlap), and therefore the artifact was not eliminated completely in the combined conductivity maps that were, however, still more accurate compared to the no-pad case.

Observing the results in Figure 6, a low dielectric but thick pad $\left(\varepsilon_{\mathrm{r}}=80, \mathrm{PT}=3 \mathrm{~cm}\right)$ and a high dielectric but thin pad $\left(\varepsilon_{\mathrm{r}}=220\right.$ or 290 , PT $\left.=1 \mathrm{~cm}\right)$ are equally most successful both in terms of $\mathrm{L}^{2}$-errors and by visual inspection. However, high dielectric and thick pads are not successful (Figure 6K, L, O, and P). For example a pad with $\varepsilon_{\mathrm{r}}=290$ and PT $=3 \mathrm{~cm}$ has high $\mathrm{L}^{2}$-error and causes gross distortions especially within the high conductivity anomaly. Adding conductivity of $\sigma=1 \mathrm{~S} / \mathrm{m}$ to the pads, the results were affected minimally when the pads were thin, whereas the errors increased with thicker pads.

To observe to what extent neglecting the derivatives of $B_{Z}$ in Equation 3 may affect the conductivity reconstructions, we did a case study where for a pad of PT $=1 \mathrm{~cm}, \varepsilon_{\mathrm{r}}=290$, and $\sigma=0 \mathrm{~S} / \mathrm{m}$, reconstruction process was repeated with the $\mathrm{B}_{\mathrm{Z}}$ terms included. The results, which are shown in Supporting Information Figure S2, were compared to reconstructions in Figure 5 that were obtained from the same pad but neglecting the $\mathrm{B}_{\mathrm{Z}}$ terms. It is observed that for the individual (not combined) cases, inclusion of the $B_{Z}$ terms significantly reduced the percent $\mathrm{L}^{2}$-errors in conductivity reconstructions. In addition, the LCF artifacts were more confined (localized) when $\mathrm{B}_{\mathrm{Z}}$ terms were included. Combined conductivities were almost of the same quality whether $B_{Z}$ terms were included or not. It appears that although neglecting the $\mathrm{B}_{\mathrm{Z}}$ terms gave rise to high error and widened LCF artifacts for the individual cases, a combination of data sets resulted in improved reconstructions even if $B_{Z}$ terms were neglected. Further simulations showed that, when $B_{Z}$ terms were neglected for high permittivity and thick pads, the combination process was not sufficient for correcting the errors that were inherent in the individual cases in which the distortions had increased more significantly. 

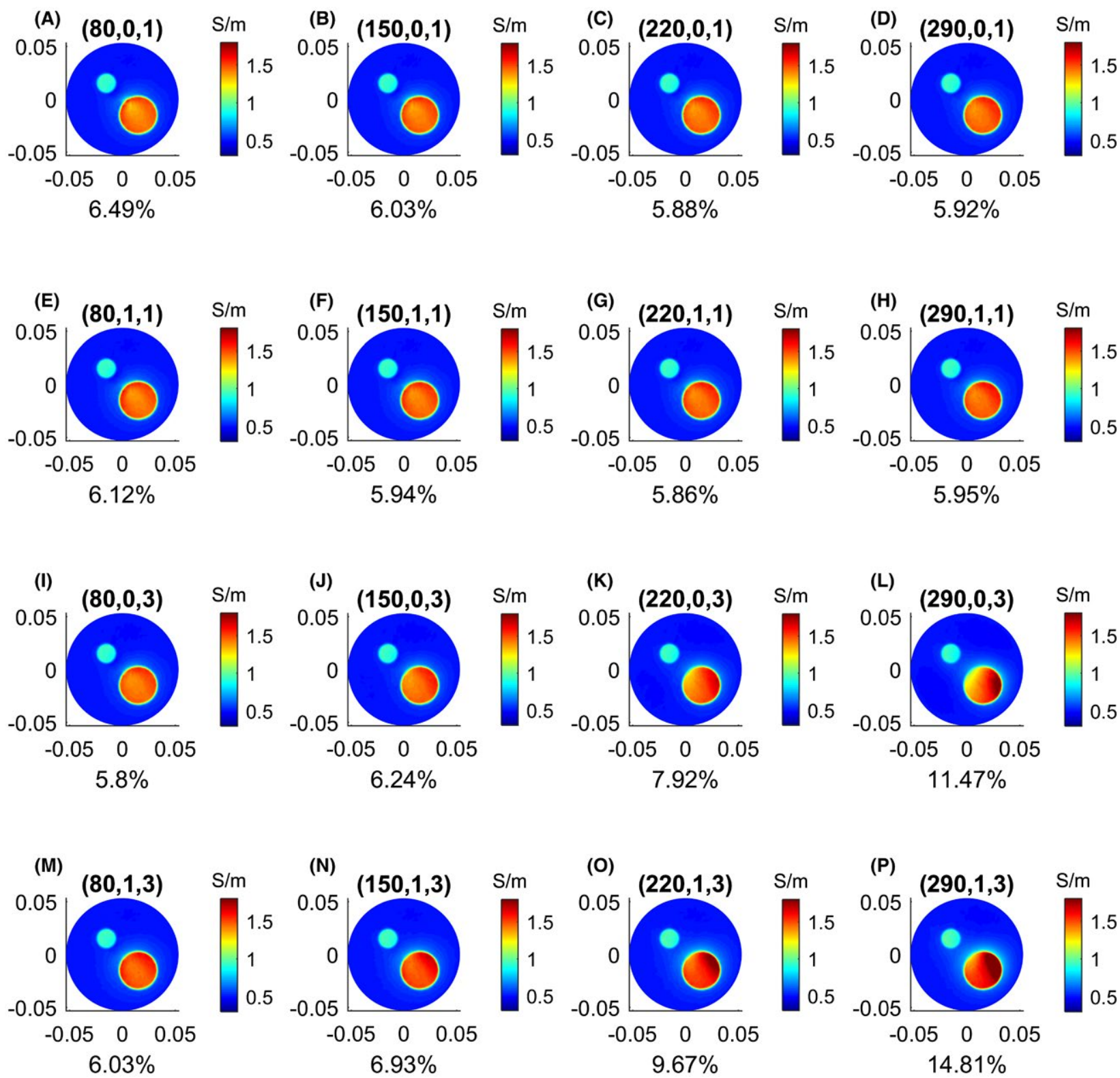

F I G U RE 6 Reconstructed conductivity images for LP + RP combinations. The properties of the pad used in each simulation are indicated as $\left(\epsilon_{\mathrm{r}}, \sigma\right.$ in $\mathrm{S} / \mathrm{m}$, pad thickness in $\left.\mathrm{cm}\right)$ above the figures, and the corresponding percent $\mathrm{L}^{2}$-errors are given below the figures

With the head phantom simulation model, the LCF artifacts in both the LP and RP cases were considerably shifted (Figure 7). The conductivity maps obtained with LP + RP and LP + NP combinations were satisfactory from the point of reduced LCF artifact, whereas with respect to conductivity values LP + NP, combination results were less erroneous on the given profile $\left(\mathrm{L}^{2}\right.$-errors for head phantom were calculated on the plotted line graphs).

\section{2 | Experimental results}

For the 2 anomaly experimental phantom, the water pad was $\sim 2.5 \mathrm{~cm}$ thick and was $\sim 2 \mathrm{~mm}$ away from the phantom (Figure 8A-C). Expected conductivity map (Figure 8D) was formed using the no-pad bSSFP magnitude image. Similar to what had been observed in simulations, the $\mathrm{B}_{1}{ }^{+}$field magnitude increased toward the pad (Figure $8 \mathrm{E}-\mathrm{G}$ ). Individual (uncombined) conductivity maps (Figure 8I-K) had severe LCF artifacts. LP + RP combined conductivity reconstruction did not suffer from LCF artifact, whereas no-pad conductivity had an LCF artifact showing itself both as a dip and a peak as seen in Figure 8M.

Conductivity maps for the no-pad case and for the LP + RP combination of other 3 different experiments are given in Figure 9. Thin water pad ( $\mathrm{PT}=1.5 \mathrm{~cm} \max )$ was not very successful at completely suppressing the LCF artifact because this pad is not thick nor high dielectric (Figure 9A-D). The resulting conductivity map, however, suffers less from 

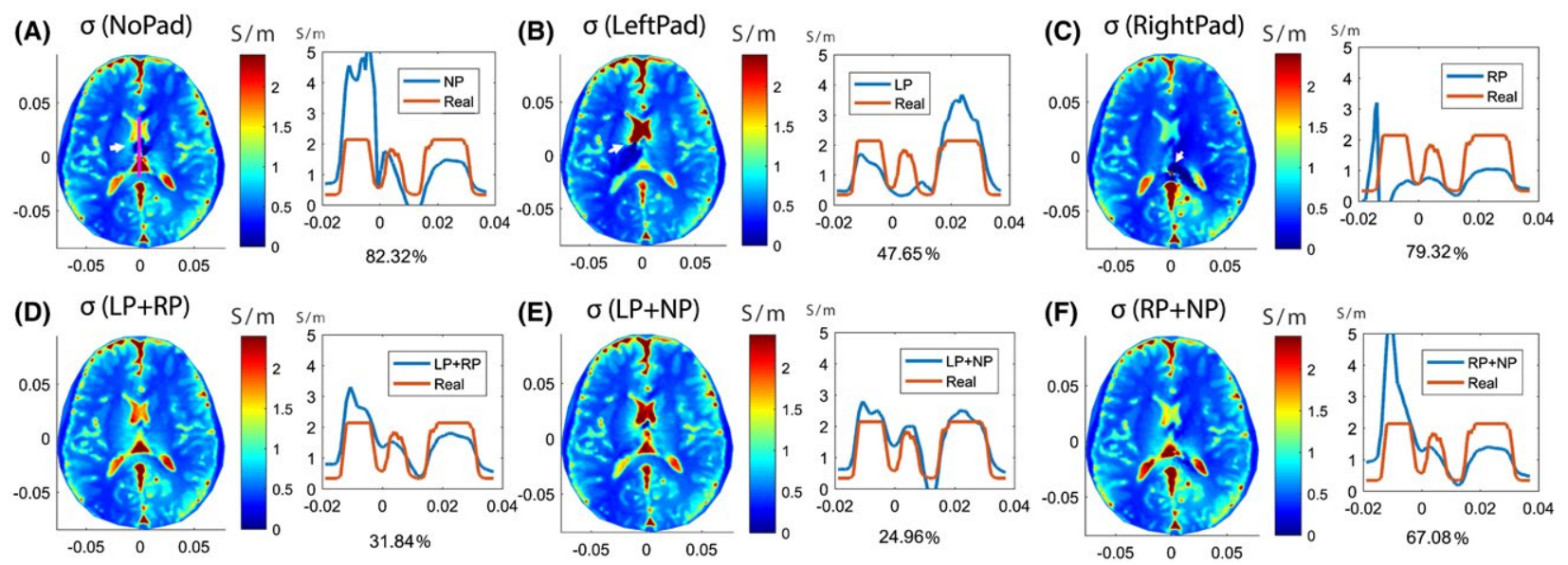

F I G U RE 7 Head phantom simulation results $\left(\mathrm{PT}=2 \mathrm{~cm}, \mathrm{GT}=2 \mathrm{~mm}, \varepsilon_{\mathrm{r}}=220\right.$ and $\left.\sigma=0 \mathrm{~S} / \mathrm{m}\right)$. The reconstructed conductivity image for (A) no-pad (NP), (B) left-pad (LP), (C) right-pad (RP), (D) LP + RP, (E) LP + NP, and (F) NP + RP. For each case, conductivity profiles on the magenta line shown in (A) are also given. LCF artifacts in (A)-(C) are indicated with white arrows. Percent $\mathrm{L}^{2}$-errors of the profiles are given below the figures
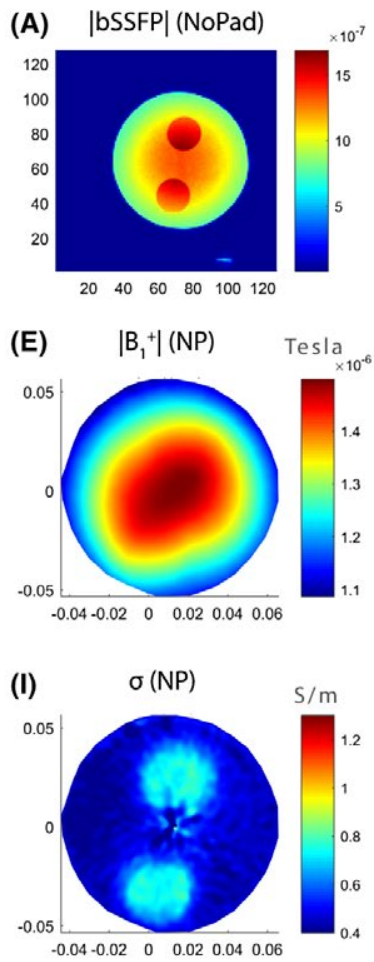
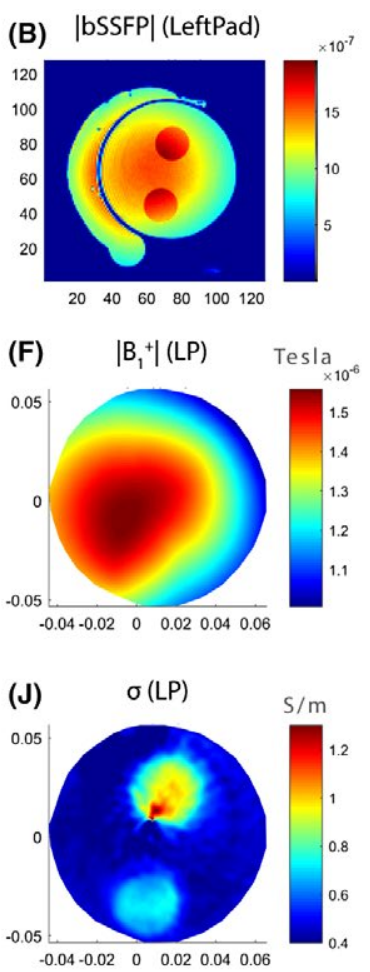
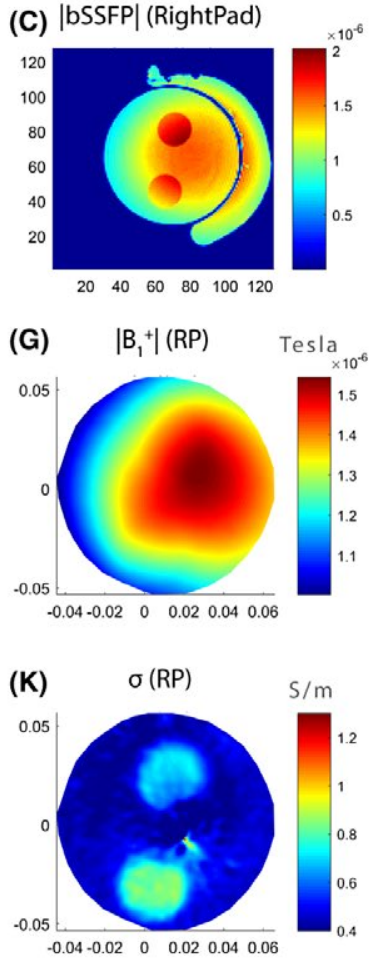

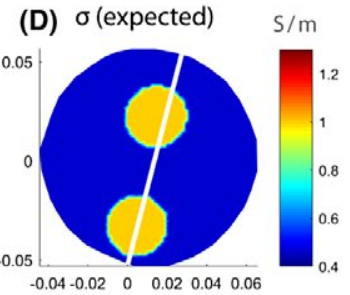

(H) $\sigma$ (std-MREPT) $\mathrm{s} / \mathrm{m}$
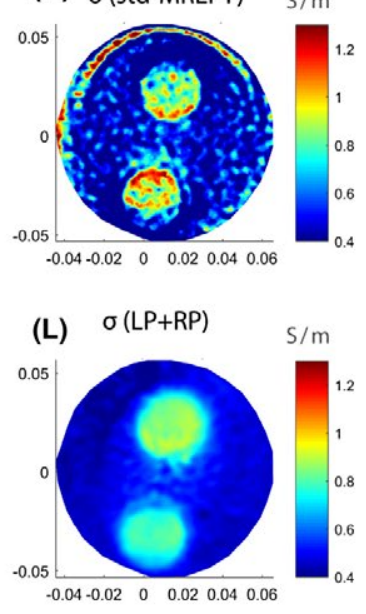

(M)

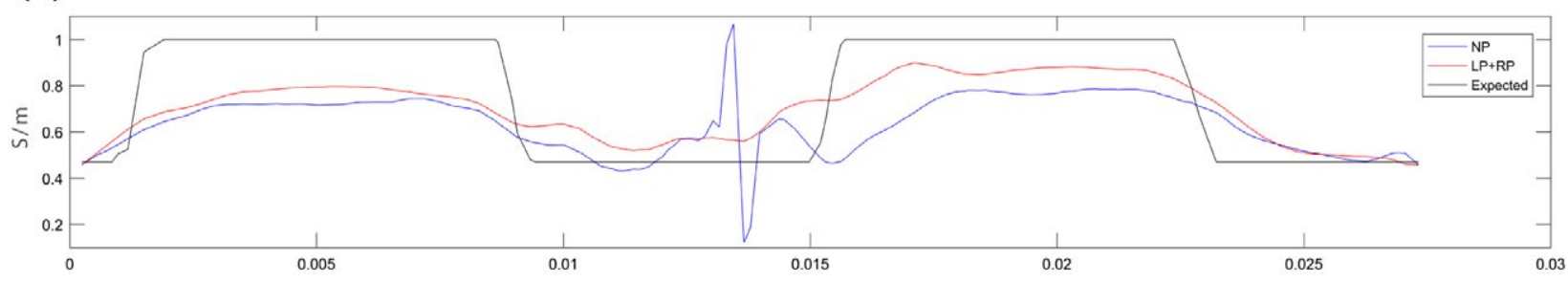

F I G U RE 8 Experimental results with a thick water pad. bSSFP magnitude images for (A) no-pad (NP), (B) left-pad (LP), and (C) right-pad (RP). (D) The expected conductivity map. B $1^{+}$magnitude maps for (E) NP, (F) left-pad (LP), and (G) right-pad (RP). (H) Conductivity image reconstructed with std-MREPT method. Reconstructed conductivity images with cr-MREPT for (I) no-pad (NP), (J) left-pad (LP), (K) right-pad (RP), and (L) LP + RP. (M) Conductivity profiles for NP and LP + RP on the line indicated in white in (D) 

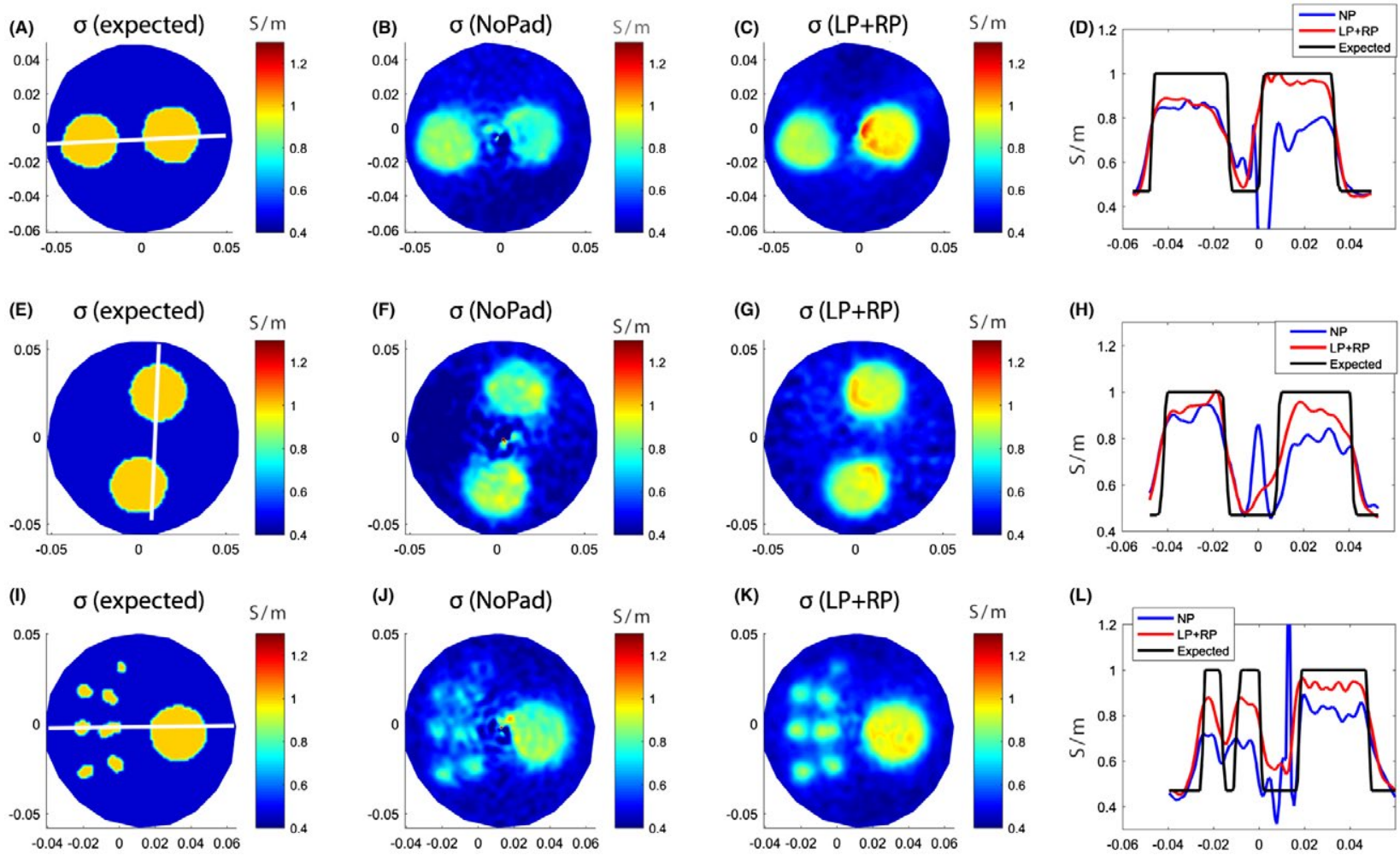

F I G U R E 9 (A-D) Results of thin water pad experiment. Expected conductivity map, reconstructed conductivity map for no-pad (NP), reconstructed conductivity map for $(\mathrm{LP}+\mathrm{RP})$, and their profiles indicated with the corresponding white line, respectively. (E-H) Same results for non-conductive $\mathrm{BaTiO}_{3}$ (Merck) pad. (I-L) Same results for conductive $\mathrm{BaTiO}_{3}$ (Entekno) pad

LCF. Using non-conductive $\mathrm{BaTiO}_{3}$ slurry pad $(\mathrm{PT}=3 \mathrm{~cm}$ max), LP + RP combined conductivity map was free from the LCF artifact, more accurate than the individual no-pad case, and the values inside the anomaly regions were in more agreement as expected (Figure 9E-H). Figure 9I-L show results for a somewhat different phantom, in which large and small anomalies were present, and for this case, conductive $\mathrm{BaTiO}_{3}$ slurry pad was used. The big anomaly region's conductivity was found to be very close to what was expected, and the LCF artifact was eliminated. Small anomalies were not easy to differentiate in the no-pad case. However, with the combined conductivity reconstruction, small anomalies were better distinguished. Furthermore, their values were very close to the big anomaly region's value as expected. Even though the simulation results with conductive pads provided less accuracy than the non-conductive ones, when the experimental results were considered, we detected no major difference between them. This was probably because of the presence of experimental noise and the filters that we applied to combat the noise. The bSSFP magnitude and the $\mathrm{B}_{1}{ }^{+}$magnitude images of the cases no-pad, the left pad, and the right pad for the experiments described in Figure 9 are given in Supporting Information Figure S3.

In additional experiments, we repeated the bSSFP sequence 32 times to achieve more averaging and therefore more SNR in the phase of $\mathrm{B}_{1}{ }^{+}$. Results of these experiments, in which a non-conductive $\mathrm{BaTiO}_{3}$ slurry pad was engaged (PT $=3 \mathrm{~cm}$ max), are shown in Figure 10. Again, LP $+\mathrm{RP}$ combinations yielded LCF artifact-free images, and the conductivity variations inside the anomaly regions themselves were decreased.

\section{DISCUSSION AND CONCLUSIONS}

cr-MREPT is a powerful electrical property mapping algorithm because it reconstructs EPs without boundary artifact, and because it is a global method, it is also robust against noise. There is, however, the inevitable LCF artifact issue where the convective field of the cr-MREPT PDE (Equation 2) is very low. In this study, the padding technique has been used to alter the transmit magnetic field distribution, $\mathrm{B}_{1}^{+}$, to obtain 2 data sets with different LCF regions. Solving the 2 linear system of equations that are composed using different data sets simultaneously, LCF artifact-free conductivity maps are acquired.

Regarding the mechanism of influence of a pad on the magnetic field in the object, it is known that dielectric and conductive pads behave differently. ${ }^{9,20,32,38}$ It has been discussed and demonstrated that according to the modified Ampere's law, displacement currents in a pad, which are 


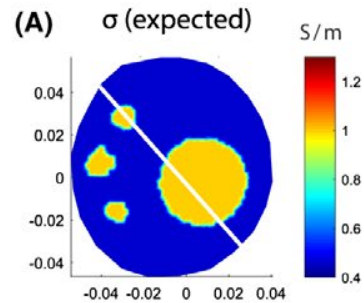

(E) $\sigma$ (expected)

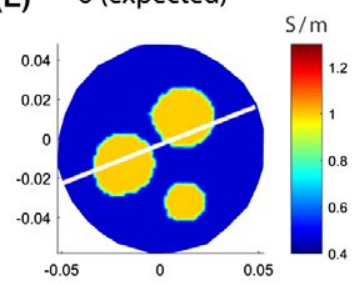

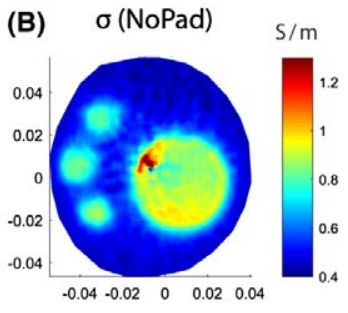

(F) $\sigma(\mathrm{NoPad})$

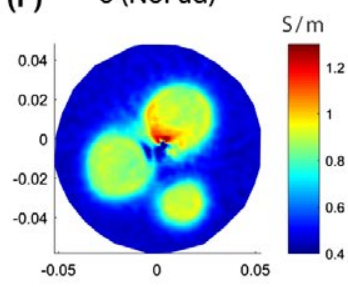

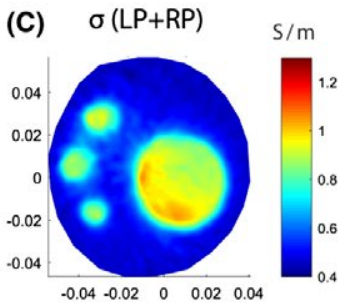
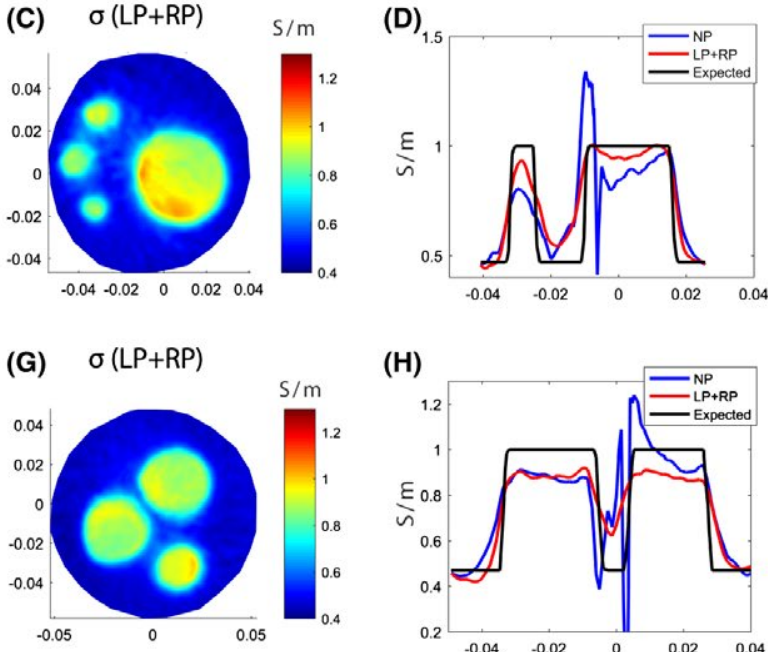

(H)

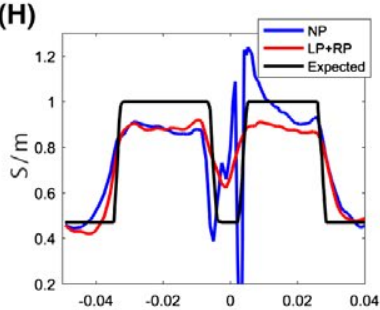

F I G URE 10 Experimental results with high SNR data and non-conductive $\mathrm{BaTiO}_{3}$ pad. (A-D) Expected conductivity map, reconstructed conductivity map for no-pad (NP), reconstructed conductivity map for LP + RP, and their profiles along the indicated white line for the 4-anomaly phantom. (E-H) Same results for the 3-anomaly phantom

induced by the "primary" $\mathrm{B}_{1}$ field of the RF coil, act as a secondary source and result in enhancement of the $\mathrm{B}_{1}$ field in regions of the object proximal to the pad. With the induced pad conduction currents (for pads with non-zero $\sigma$ ), which are $90^{\circ}$ out of phase with the displacement currents, such enhancement is not realized, and the $\mathrm{B}_{1}$ field is modified differently. Our simulation results in Figure 2 are consistent with these findings. We have also observed differential effects of pad electrical properties on the LCF shifts. As seen in Figure $3 \mathrm{D}$, for a specific placement of the pure dielectric pad, the LCF shift is always in a specific direction toward the pad, and one can change the pad parameters to adjust the amount of the shift along that same direction. On the other hand, for pads with both dielectric and conductive properties, varying the conductivity changes the direction of the LCF shift as shown in Figure 3E.

Analyses of the pad parameters, which provide essential information on how to adapt the pad in practice, are illustrated in Figure 4. Increasing the dielectric constant of the pad and/or the pad thickness increases the amount of LCF shift. Increased gap thickness, on the other hand, results in less LCF shift. Increasing the angle of the arc that the pad subtends also increases the shift, and yet, covering more than half of the phantom is not beneficial. It is also demonstrated that using a pad whose height is the same as the object's, yields the most effective shift.

Conductivity reconstructions obtained for the cylindrical simulation phantom with 2 anomaly regions (Figures 5 and 6 and Supporting Information Figure S1) demonstrated that combining 2 data sets with different pad conditions, and therefore with different LCF locations, succeeds in reducing the LCF artifact. LCF regions were maximally shifted away from each other for pads in opposite sides of the object, and combining data from them could result in almost complete elimination of the LCF artifact as revealed in terms of percent $\mathrm{L}^{2}$-error as well as by visual inspection of the images and profile plots. It was also demonstrated that although the amount of the LCF shift is important so that the LCF regions do not overlap, excessive amount of shift is undesired. In this context, high dielectric but thin pads (e.g. $\varepsilon_{\mathrm{r}}=290, \mathrm{PT}=1 \mathrm{~cm}$ ) or thick water pads $\left(\varepsilon_{\mathrm{r}}=80, \mathrm{PT}=3 \mathrm{~cm}\right)$ performed the best (Figure 6). Further simulations also showed that neglecting the $\mathrm{B}_{Z}$ derivatives in the convective field defined in Equation 3 may be the reason for low performance with pads of both high dielectric constant and high thickness. Another observation derived from Figure 6 is that adding conductivity to a pad, which already has at least a relative permittivity of 80 because of its water content, makes no significant improvement on the conductivity maps.

Simulation results of a head phantom (Figure 7) also demonstrated that with the padding technique, the LCF artifact was reduced. The individual conductivity reconstruction of the head phantom with right-sided pad placement produced a poor conductivity map, indicating that this pad configuration may not be an optimum one for this phantom. Although the final combined conductivity maps provide more accurate images, still further inspection on the optimum pad configuration is needed for a complex EP distribution such as in the head.

Experimental results (Figures 8-10) corroborate the findings in simulation studies. Additionally, it was found that the advantage of the padding technique becomes even more pronounced in the noisier experiments such that the conductivity images are relatively more significantly improved (e.g., although the small anomalies in Figure 9J were almost undetectable, in the combined conductivity map in Figure 9K, all anomaly regions could be distinguished). The conductivities of the anomaly 
regions were found to be less than the expected values that were determined based on the concentrations of $\mathrm{NaCl}, \mathrm{CuSO}_{4}$, and agar used in the phantom solutions and gels as explained in Hafalir et al. ${ }^{10}$ Temperature difference between the phantom preparation room and the cool scanner room, diffusion of $\mathrm{NaCl}$ during long data acquisition periods, and structural change in agar gel during storage may have caused alterations in the expected values of conductivity up to 10-20\%. Therefore, results of experimental studies are to be evaluated not in terms of absolute conductivity accuracy but rather with respect to elimination of the LCF artifact that is the main theme of this study.

Throughout the article, reconstructions obtained by combining only 2 data sets are provided. This emanates from the foresight that more MR scans would bring additional time cost. Furthermore, incorporation of a third data set into the combination may not always yield better results. It is depicted that $180^{\circ}$ pad arc maintains the largest LCF shift and 2 oppositely placed pads give the furthest away LCF locations. However, an additional pad location may lead to the overlapping of the LCF regions causing LCF artifacts to reappear. However, in more complex objects such as the brain, different pad combinations may be optimal in different regions.

With the multiple data acquisition requirement of the padding method, subject movement between scans is unavoidable. This may be because of the involuntary movements of the subjects in between scans, or because of the dislocation of the subject while placing the pad or altering its position. In either case, a registration problem arises. We have suggested using a genetic algorithm-based registration, in which the edge information from the bSSFP magnitude images are collected, and the algorithm is run until the edges match each other. Simulation studies reported in Supporting Information Figure S4 aim at determining to what extent this method is successful. The case with the right pad is kept fixed, and for the case with left pad, both the object and the pad are shifted and rotated. For different shift and rotation amounts, LP + RP combined conductivity images are formed with and without registration. It is found that, for $<1 \mathrm{~mm}$ dislocations image distortions because of lack of registration are not very detrimental. For $2 \mathrm{~mm}$ shifts, however, image distortions are significant if registration is not applied, based on visual inspection and also by observing the percent $\mathrm{L}^{2}$-errors. Fortunately, with registration, almost full recovery can be achieved. In conclusion, with a registration algorithm, as long as shifts are reduced to the $1 \mathrm{~mm}$ range, distortions may be corrected to a large extent. $\mathrm{L}^{2}$-errors of $1 \mathrm{~mm}$ and $2 \mathrm{~mm}$ shifted and corrected images are less than the $\mathrm{L}^{2}$-error of $0.5 \mathrm{~mm}$ shifted and corrected image, because the data were acquired with $1 \mathrm{~mm}$ resolution and interpolation of data with shifts with integer multiples of $1 \mathrm{~mm}$ is performed more accurately than interpolation of data with sub-pixel shift. There is also the problem of motion artifact that arises because of movement during a scan itself. This issue, however, is very difficult to resolve with registration.
The best measure to take is to shorten the required scan time. Although we have used the double angle method to obtain $\mathrm{B}_{1}{ }^{+}$ magnitude, Bloch-Siegert shift ${ }^{39}$ and DREAM ${ }^{40}$ methods provide faster maps; the latter method, on the other hand, requires additional noise improvement. For phase imaging, bSSFP sequence gives rather high SNR within a tolerable time interval.

The proposed padding method may be beneficial, not only for the cr-MREPT algorithm, but also for other MREPT methods as well. In general, methods that do not neglect the gradient term in Equation 2 experience the LCF artifact. Many investigators have, in fact, reported this artifact although they may not have named it the same. With certain assumptions, the convective field can be expressed as $\mathrm{F}_{\mathrm{x}}=\frac{\partial \mathrm{B}_{1}^{+}}{\partial \mathrm{x}}-\mathrm{i} \frac{\partial \mathrm{B}_{1}^{+}}{\partial \mathrm{y}}=\frac{\mathrm{i}}{2} \mu_{0} \mathrm{E}_{\mathrm{Z}} \gamma$ where $\mathrm{E}_{\mathrm{z}}$ is the axial electric field. Because $\gamma \neq 0$, it follows that if the convective field is 0 , $\mathrm{E}_{\mathrm{z}}$ is also 0. In Fushimi and Nara ${ }^{14}$ and Nara et al., ${ }^{15}$ a 2-step algorithm for MREPT has been proposed and implemented, where they first estimate $\mathrm{E}_{\mathrm{z}}$ from the knowledge of $\mathrm{B}_{1}{ }^{+}$and

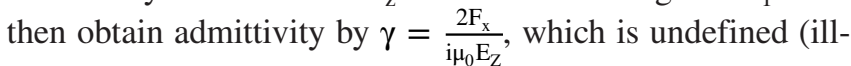
defined) in the LCF region. They have recognized the fact that in the low electric field region there is a spot-like artifact, and with additive noise it is more prominent and widened. They have, in fact, acknowledged the proposal made in Hafalir et al. ${ }^{10}$ that pads may be used to displace the low electric field region, and thereby padding may benefit their algorithm as well. Ammari et al. ${ }^{13}$ use a similar PDE to solve for $\gamma$ and they realize that in regions where the convective field is 0 (or low), their matrix becomes degenerate (or ill-conditioned), and therefore they use std-MREPT in those regions as is originally proposed in Hafalir et al. ${ }^{10}$ as an interim remedy. They also use their solution as an initial estimate for their inverse approach called the "adjoint method" and iterate to approach the measured $\mathrm{B}_{1}{ }^{+}$. In this "inverse problem approach" they minimize a functional that involves the norm of the difference between the calculated $\mathrm{B}_{1}{ }^{+}$and the measured $\mathrm{B}_{1}{ }^{+}$. It is easy to modify this functional to have the sum of the least square errors from 2 experiments, with pad and without pad, and therefore probably approach a better solution. Li et al. ${ }^{28}$ have also recognized, in reference to Hafalir et al., ${ }^{10}$ that in regions where $\left|F_{X}\right|$ is low, there is an artifact in their reconstructions. They attempt to solve the problem by introducing a diffusion term in the cr-MREPT PDE as regularization. They mention, however, that it is not sufficient under noise. It is also possible to introduce 2 sets of data into their algorithm (i.e. solve the 2 systems simultaneously). Liu et al. ${ }^{17}$ have experienced and stated that in regions where $\left|F_{X}\right|$ is low, a "global bias" occurs in their conductivity reconstructions obtained by the gEPT method. This method already solves multiple equations, derived from data obtained via multichannel RF coils, and using more equations acquired with pad placement would increase its accuracy. ${ }^{11}$ Padding has already been adapted to a CSIbased method, ${ }^{18}$ and a significant improvement has been 
recorded. Although this method combines the maps by averaging them, simultaneously solving their linear system of equations may even give improved results.

Schmidt and Webb ${ }^{18}$ have been the first investigators who have suggested the use of padding to improve their CSI-based MREPT method. Their study was based on simulations only and they used a single pad structure. We believe some of the additional contributions of the present work are demonstration of the use of padding in the context of another algorithm (i.e., cr-MREPT), analysis of the mechanism of pad effect, analysis of the effects of pad parameters, analysis of the importance of neglecting $\mathrm{B}_{\mathrm{z}}$, implementation of padding also in real phantom experiments, and simultaneous use of data from different pad cases by concatenating the corresponding sets of equations rather than averaging the individual conductivities reconstructed from individual pad cases. Finally, in parallel with our observations, Schmidt and Webb ${ }^{18}$ have anticipated that using very high permittivity values (such as 500) causes significant artifacts even at 3T, and lower permittivity pads would need to be used for higher field implementations.

Regularization methods have been proposed by several investigators to reduce noise effects and/or algorithm-caused spurious oscillations. ${ }^{28,41,42}$ As mentioned in the introduction, artificial diffusion, which is another way of regularization, is proposed for reduction of the LCF artifact. ${ }^{28,29}$ In this work, we have not used any regularization method because we wanted to concentrate on the performance and limitations of the padding technique.

In conclusion, this study provides artifact-free conductivity reconstruction by improving the so called cr-MREPT algorithm with the use of dielectric pads. Several parameters that determine the pad behavior and the resulting reconstructions are analyzed to obtain the optimum padding set-up. Future work may cover combined use of regularization and padding as well as comparison of the 2 approaches and investigating the optimum pad structures for more complex phantoms.

\section{ACKNOWLEDGMENT}

This study was supported by TUBITAK 114E522 research grant. Experimental data were acquired using the facilities of UMRAM, Bilkent University, Ankara.

\section{ORCID}

Gulsah Yildiz (D) https://orcid.org/0000-0002-2082-0458
Yusuf Ziya Ider (D) http://orcid.org/0000-0002-1961-6804

\section{REFERENCES}

1. Joines W, Zhang Y, Li C, Jirtle R. The measured electrical properties of normal and malignant human tissues from 50 to $900 \mathrm{MHz}$. Med Phys. 1994;21:547-550.
2. Surowiec A, Stuchly S, Barr J, Swarup A. Dielectric properties of breast carcinoma and the surrounding tissues. IEEE Trans Biomed Eng. 1988;35:257-263.

3. Katscher U, Voigt T, Findeklee C, Vernickel P, Nehrke K, Dossel O. Determination of electric conductivity and local SAR via B1 mapping. IEEE Trans Med Imaging. 2009;28:1365-1374.

4. Salinas F, Lancaster J, Fox P. 3D modeling of the total electric field induced by transcranial magnetic stimulation using the boundary element method. Phys Med Biol. 2009;54:3631-3647.

5. Balidemaj E, Kok H, Schooneveldt G, et al. Hyperthermia treatment planning for cervical cancer patients based on electrical conductivity tissue properties acquired in vivo with EPT at 3 T MRI. Int J Hyperthermia. 2016;32:558-568.

6. Kwon O, Chauhan M, Kim H, et al. Fast conductivity imaging in magnetic resonance electrical impedance tomography (MREIT) for RF ablation monitoring. Int J Hyperthermia. 2014;30:447-455.

7. Haacke E, Petropoulos L, Nilges E, Wu D. Extraction of conductivity and permittivity using magnetic resonance imaging. Phys Med Biol. 1991;36:723-734.

8. Wen H. Noninvasive quantitative mapping of conductivity and dielectric distributions using RF wave propagation effects in highfield MRI. In Proceedings of the Conference on Medical Imaging, Physics of Medical Imaging, San Diego, CA, 2003. Volume 5030.

9. Vaidya M, Collins C, Sodickson D, Brown R, Wiggins G, Lattanzi R. Dependence of B1- and B1+ field patterns of surface coils on the electrical properties of the sample and the MR operating frequency. Concepts Magn Reson Part B Magn Reson Eng. 2016;46:25-40.

10. Hafalir F, Oran O, Gurler N, Ider Y. Convection-reaction equation based magnetic resonance electrical properties tomography (crMREPT). IEEE Trans Med Imaging. 2014;33:777-793.

11. Liu J, Zhang X, Schmitter S, Van de Moortele P, He B. Gradientbased electrical properties tomography (gEPT): a robust method for mapping electrical properties of biological tissues in vivo using magnetic resonance imaging. Magn Reson Med. 2014;74:634-646.

12. Balidemaj E, van den Berg C, Trinks J, et al. CSI-EPT: a contrast source inversion approach for improved MRI-based electric properties tomography. IEEE Trans Med Imaging. 2015;34:1788-1796.

13. Ammari H, Kwon H, Lee Y, Kang K, Seo J. Magnetic resonancebased reconstruction method of conductivity and permittivity distributions at the Larmor frequency. Inverse Probl. 2015;31:105001.

14. Fushimi M, Nara T. A boundary-value-free reconstruction method for magnetic resonance electrical properties tomography based on the Neumann-type integral formula over a circular region. SICE J Control Meas Syst Integr. 2017;10:571-578.

15. Nara T, Furuichi T, Fushimi M. An explicit reconstruction method for magnetic resonance electrical property tomography based on the generalized Cauchy formula. Inverse Probl. 2017;33:105005.

16. Ariturk G, Ider Y. Optimal multichannel transmission for improved cr-MREPT. Phys Med Biol. 2018;63:045001.

17. Liu J, Wang Y, Katscher U, He B. Electrical properties tomography based on B1 maps in MRI: principles, applications, and challenges. IEEE Trans Biomed Eng. 2017;64:2515-2530.

18. Schmidt R, Webb A. A new approach for electrical properties estimation using a global integral equation and improvements using high permittivity materials. J Magn Reson. 2016;262:8-14.

19. Zhang X, Zhu S, He B. Imaging electric properties of biological tissues by RF field mapping in MRI. IEEE Trans Med Imaging. 2010;29:474-481. 
20. Yang Q, Wang J, Wang J, Collins C, Wang C, Smith M. Reducing SAR and enhancing cerebral signal-to-noise ratio with high permittivity padding at 3 T. Magn Reson Med. 2010;65:358-362.

21. Yang Q, Herse Z, Ketterman M, et al. Enhancement of RF field by high dielectric constant pad at 3T: cervical spine imaging. In Proceedings of the 19th Annual Meeting of ISMRM, Montreal, Canada, 2011. p. 621.

22. Schmitt M, Feiweier T, Horger W, et al. Improved uniformity of RF-distribution in clinical whole body- imaging at $3 \mathrm{~T}$ by means of dielectric pads. In Proceedings of the 12th Annual Meeting of ISMRM, Kyoto, Japan, 2004. p. 197.

23. Haines K, Smith N, Webb A. New high dielectric constant materials for tailoring the $\mathrm{B} 1+$ distribution at high magnetic fields. $J$ Magn Reson. 2010;203:323-327.

24. Yildiz G, Ariturk G, Ider YZ. Use of padding to eliminate the LCF artifact in conductivity maps obtained by cr-MREPT. In Proceedings of the 25th Annual Meeting of ISMRM, Honolulu, HI, 2017. Abstract 1949.

25. Gurler N, Ider Y. Gradient-based electrical conductivity imaging using MR phase. Magn Reson Med. 2016;77:137-150.

26. van Lier A, Brunner D, Pruessmann K, et al. B1+ phase mapping at $7 \mathrm{~T}$ and its application for in vivo electrical conductivity mapping. Magn Reson Med. 2011;67:552-561.

27. Voigt T, Katscher U, Doessel O. Quantitative conductivity and permittivity imaging of the human brain using electric properties tomography. Magn Reson Med. 2011;66:456-466.

28. Li C, Yu W, Huang S. An MR-based viscosity-type regularization method for electrical property tomography. Tomography. 2017;3:50-59.

29. Ider YZ, Boga C. Spatial resolution of Full cr-MREPT: 2D and 3D evaluation. In Proceedings of the 26th Annual Meeting of ISMRM, Paris, France, 2018. Abstract 5098.

30. Gurler N, Ider Y. Numerical methods and software tools for simulation, design, and resonant mode analysis of radio frequency birdcage coils used in MRI. Concepts Magn Reson Part B Magn Reson Eng. 2015;45:13-32.

31. Iwase E. Electrical conductivities of the salt solutions containing agar. Bull Chem Soc Jpn. 1927;2:61-65.

32. Webb A. Dielectric materials in magnetic resonance. Concepts Magn Reson Part A. 2011;38A:148-184.

33. Luo W, Lanagan M, Sica C, et al. Permittivity and performance of dielectric pads with sintered ceramic beads in MRI: early experiments and simulations at 3 T. Magn Reson Med. 2012;70:269-275.

34. Meskine F, Mezouar M, Taleb N. A rigid image registration based on the nonsubsampled contourlet transform and genetic algorithms. Sensors. 2010;10:8553-8571.

35. Stollberger R, Wach P. Imaging of the active B1 field in vivo. Magn Reson Med. 1996;35:246-251.

36. Stehning C, Voigt TR, Katscher U. Real-time conductivity mapping using balanced SSFP and phase-based reconstruction. In Proceedings of the 19th Annual Meeting of ISMRM, Montreal, Canada. 2011. p. 128.

37. Fernandez FA, Kulas L. A simple finite difference approach using unstructured meshes from FEM mesh generators. In Proceedings of IEEE 15th International Conference on Microwaves, Radar and Wireless Communications, Warsaw, Poland, 2004. p. 585-588.
38. Yang Q, Mao W, Wang J, et al. Manipulation of image intensity distribution at $7.0 \mathrm{~T}$ : passive RF shimming and focusing with dielectric materials. J Magn Reson Imaging. 2006;24: 197-202.

39. Sacolick L, Wiesinger F, Hancu I, Vogel M. B1 mapping by BlochSiegert shift. Magn Reson Med. 2010;63:1315-1322.

40. Nehrke K, Börnert P. DREAM-a novel approach for robust, ultrafast, multislice B1 mapping. Magn Reson Med. 2012;68:1517-1526.

41. Ropella K, Noll D. A regularized, model-based approach to phase-based conductivity mapping using MRI. Magn Reson Med. 2017;78:2011-2021.

42. Wang Y, Van de Moortele P, He B. CONtrast conformed electrical properties tomography (CONCEPT) based on multi-channel transmission. In Proceedings of the 25th Annual Meeting ISMRM, Honolulu, HI, 2017. Abstract 3640.

\section{SUPPORTING INFORMATION}

Additional supporting information may be found online in the Supporting Information section at the end of the article.

FIGURE S1 Simulation results with the $\epsilon_{\mathrm{r}}=80, \sigma=0 \frac{\mathrm{s}}{\mathrm{m}}$, pad thickness $=1 \mathrm{~cm}$, and gap thickness $=2 \mathrm{~mm}$ pad. (A-C) Magnitude of $\mathrm{B}_{1}{ }^{+}$. (D-F) Magnitude of $\mathrm{F}_{\mathrm{X}}$. (G-I) Reconstructed conductivity image for left-pad (LP), no-pad (NP), and right-pad (RP), respectively. (J) Reconstructed conductivity image for (LP $+\mathrm{NP}$ ) combination. (K) Reconstructed conductivity image for $(\mathrm{RP}+\mathrm{NP})$ combination. ( L) Reconstructed conductivity image for (LP + RP) combination. (M) Real conductivity map. (N) Conductivity profiles on the line given in $(\mathrm{M})$. Percent $\mathrm{L}^{2}$-errors are given below the figures

FIGURE S2 Conductivity reconstructions using Equation 3 , which includes the $\mathrm{B}_{\mathrm{Z}}$ derivatives, for the pad and the phantom used in Figure 5. Conductivity images for (A) left-pad (LP), (B) no-pad (NP), (C) right-pad (RP), (D) (LP $+\mathrm{NP}),(\mathrm{E})(\mathrm{RP}+\mathrm{NP})$, and (F) (LP + RP). Pad parameters are: $\epsilon=290, \sigma=0 \mathrm{~S} / \mathrm{m}$, pad thickness $=1 \mathrm{~cm}$, gap thickness $=2 \mathrm{~mm}$

FIGURE S3 bSSFP magnitude images and $\mathrm{B}_{1}{ }^{+}$magnitude images for the experiments given in Figure 9. (A-F) Thin water pad. (G-L) Non-conductive $\mathrm{BaTiO}_{3}$ (Merck) pad. (M-R) Conductive $\mathrm{BaTiO}_{3}$ (Entekno) pad

FIGURE S4 Effect of the object movement between scans. The case with the right pad is kept fixed, and both the object and the pad is shifted and rotated for the case with left pad. For different shift and rotation amounts: first column, the individual conductivity map for left pad (LP) case without any corrections; second column, projections of the edge information of the left and the right pad cases onto each other (green edge, RP; purple edge, LP; black edge, both RP and 
LP); third column, LP + RP combined conductivity maps when no correction is applied; and fourth column, LP + RP combined conductivity maps when the proposed correction is applied. Percent $\mathrm{L}_{2}$-errors for each conductivity map is given below the figures. The numbers below the projections indicate the squared difference between the 2 projections by defining the value of the edge information as 1 and the rest as 0 . Pad thickness $=3 \mathrm{~cm}$, gap thickness $=2 \mathrm{~mm}, \varepsilon=150$, $\sigma=0 \mathrm{~S} / \mathrm{m}$
VIDEO S1 Animation showing the rotating current distribution in the pad for the simulation described in Figure 2

How to cite this article: Yildiz G, Ider YZ. Use of dielectric padding to eliminate low convective field artifact in cr-MREPT conductivity images. Magn Reson Med. 2019;81:3168-3184. https://doi. org $/ 10.1002 / \mathrm{mrm} .27648$ 